\title{
Symptoms and the body: Taking the inferential leap
}

Article in Neuroscience \& Biobehavioral Reviews · January 2017

DOI: 10.1016/j.neubiorev.2017.01.015

CITATIONS

0

4 authors, including:

\section{Omer Van den Bergh}

University of Leuven

282 PUBLICATIONS 4,738 CITATIONS

SEE PROFILE

Sibylle Petersen

University of Leuven

36 PUBLICATIONS 195 CITATIONS

SEE PROFILE

\section{READS}

121

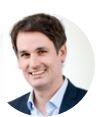

Michael Witthöft

Johannes Gutenberg-Universität Mainz 109 PUBLICATIONS 1,204 CITATIONS

SEE PROFILE

Some of the authors of this publication are also working on these related projects:

Project
Development and Evaluation of a specific cognitive behavioral therapy program for migraine in adults View project

Memory processes in retrospective symptom (over)reporting View project 
Review article

\title{
Symptoms and the body: Taking the inferential leap
}

\author{
Omer Van den Bergh $^{\mathrm{a}, *}$, Michael Witthöft $^{\mathrm{b}}$, Sibylle Petersen ${ }^{\mathrm{a}, \mathrm{c}}$, Richard J. Brown $^{\mathrm{d}, \mathrm{e}}$ \\ a Health Psychology, University of Leuven, Tiensestraat 102, Belgium \\ b Clinical Psychology, Psychotherapy, and Experimental Psychopathology, University of Mainz, Germany \\ ${ }^{\mathrm{c}}$ Institute for Health and Behaviour, University of Luxembourg, Luxembourg and Health Psychology, University of Leuven, Belgium \\ d Centre for New Treatments and Understanding in Mental Health, Division of Psychology and Mental Health, School of Health Sciences, Faculty of Biology, \\ Medicine and Health, University of Manchester, Manchester Academic Health Sciences Centre, UK \\ e Psychological Services, Greater Manchester Mental Health NHS Foundation Trust, Manchester Academic Health Sciences Centre, UK
}

\section{A R T I C L E I N F O}

\section{Article history:}

Received 12 August 2016

Received in revised form 1 December 2016

Accepted 11 January 2017

Available online 17 January 2017

\section{Keywords:}

Symptom perception

Medically unexplained symptoms

Predictive coding

\begin{abstract}
A B S T R A C T
The relationship between the conscious experience of physical symptoms and indicators of objective physiological dysfunction is highly variable and depends on characteristics of the person, the context and their interaction. This relationship often breaks down entirely in the case of "medically unexplained" or functional somatic symptoms, violating the basic assumption in medicine that physical symptoms have physiological causes. In this paper, we describe the prevailing theoretical approach to this problem and review the evidence pertaining to it. We then use the framework of predictive coding to propose a new and more comprehensive model of the body-symptom relationship that integrates existing concepts within a unifying framework that addresses many of the shortcomings of current theory. We describe the conditions under which a close correspondence between the experience of symptoms and objective physiology might be expected, and when they are likely to diverge. We conclude by exploring some theoretical and clinical implications of this new account.
\end{abstract}

(c) 2017 Elsevier Ltd. All rights reserved.

\section{Contents}

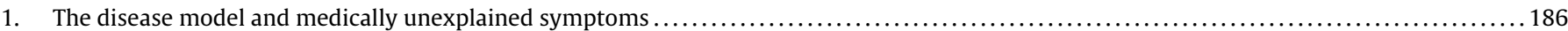

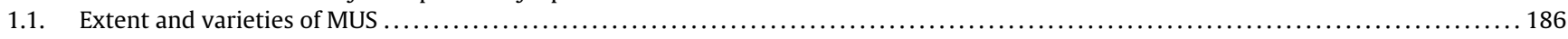

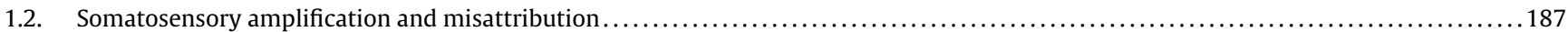

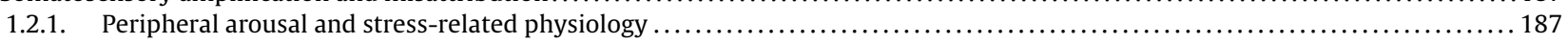

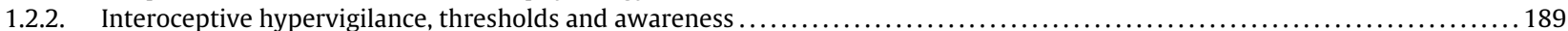

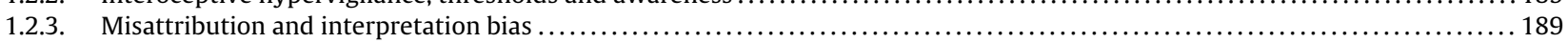

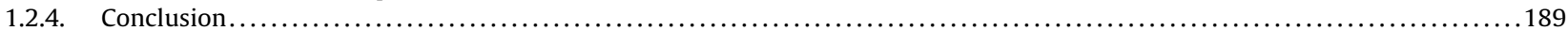

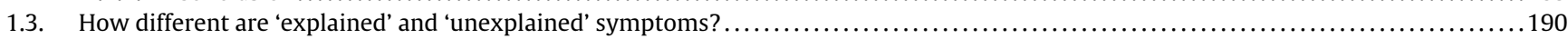

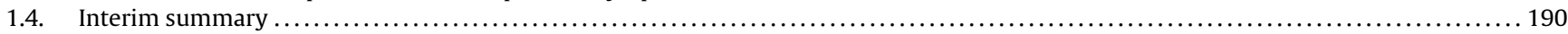

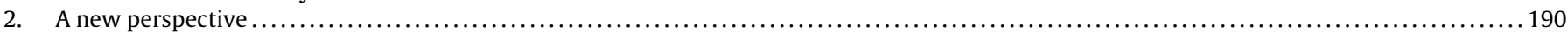

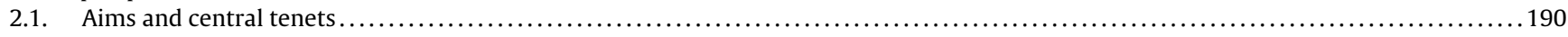

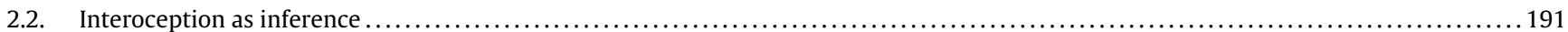

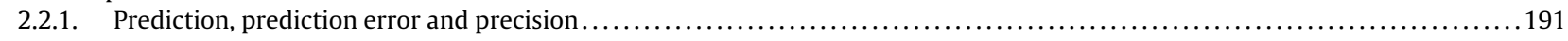

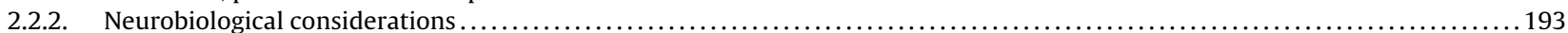

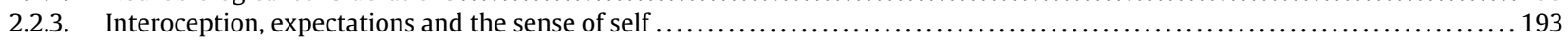

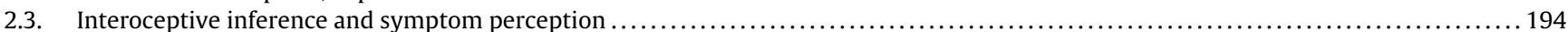

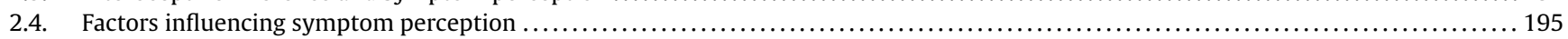

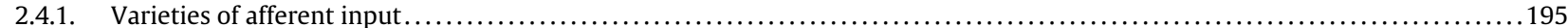

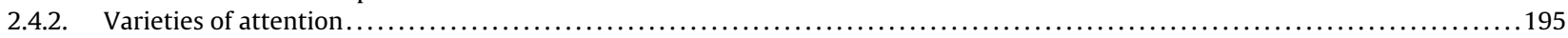

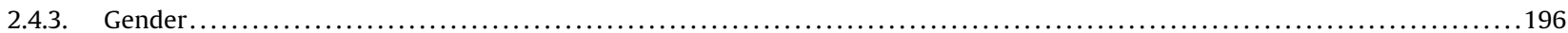

\footnotetext{
* Corresponding author.

E-mail address: omer.vandenbergh@kuleuven.be (O. Van den Bergh).
} 


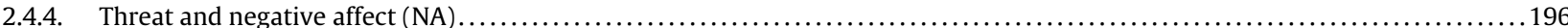

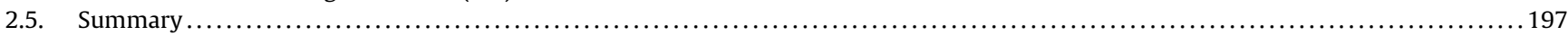

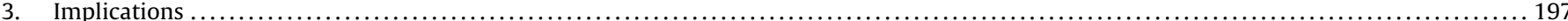

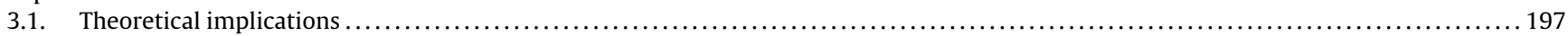

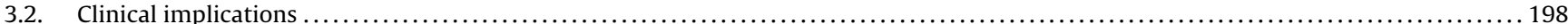

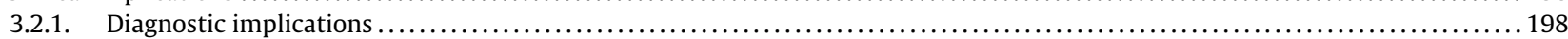

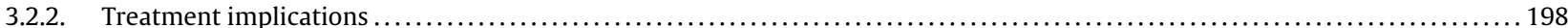

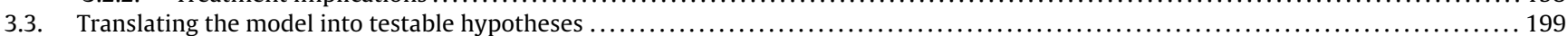

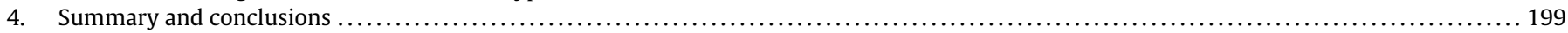

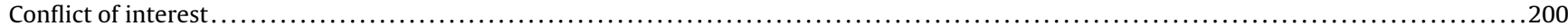

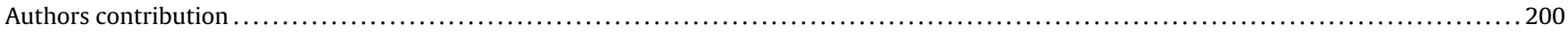

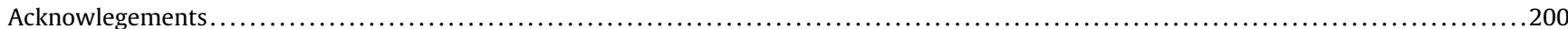

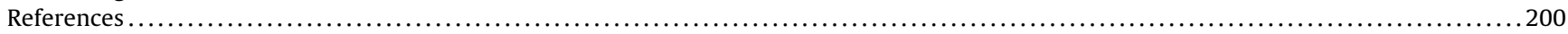

\section{The disease model and medically unexplained symptoms}

Standard medical practice is premised on a disease model that typically comprises two phases. The diagnostic phase begins when symptoms are reported to a physician, who looks to determine their cause through history taking, physical examination and, where appropriate, medical investigations. This information, which mainly concerns the patient's body, is mapped onto a set of pathophysiological criteria that allow for diagnosis and treatment. In the therapeutic phase, the aim is to remedy dysfunction and thereby remove the patient's symptoms.

This apparently logical process is often successful, but it sometimes fails dramatically. A particularly compelling (and common) example of this is when the patient reports symptoms despite tests indicating that their body is healthy, or where "successful" treatment for diagnosed disease fails to resolve symptoms. In such cases, doctors often make renewed attempts to identify disease, reflecting one of the fundamental assumptions of this model: that physical symptoms have physiological causes, and can therefore be reduced to them. If the symptoms persist but a disease cause remains elusive, then the patient may be given a diagnosis that simply describes their complaint (e.g., chronic fatigue) or another label that identifies them as suffering from "medically unexplained symptoms" (MUS). Although the biopsychosocial model has ensured that symptoms are no longer seen as purely biological phenomena, medical practice continues to be dominated by the view that "real" symptoms reflect bodily dysfunction, and that those symptoms that cannot be validated objectively are "in the mind" or simply made up.

In this paper, we draw on previous approaches to develop a novel model of symptom perception that transcends the artificial distinction between "explained" and "unexplained" physical symptoms, whilst explaining the variable relationship between symptoms and physiological dysfunction. The central principle underpinning this account is that physical symptoms, as felt and expressed by patients, are not a direct record of bodily activity, but an inference based on implicit predictions about interoceptive information, derived from prior knowledge. An important implication of this account is that symptoms often result from an "inferential leap", resulting in an experience that is only loosely coupled with dysfunctional processes in the peripheral body, and occasionally has no relationship at all. We use this framework to describe the conditions under which a close correspondence between subjective symptoms and objective physiology might be expected, and when the two are likely to diverge. We conclude by exploring some clinical and empirical implications.

\subsection{Extent and varieties of MUS}

Physical symptoms that occur in the absence of detectable physiological dysfunction are ubiquitous. In a population-based study in Germany ( $N=2552)$, for example, $81.6 \%$ of people reported at least one medically unexplained symptom causing at least mild impairment (Hiller et al., 2006). In primary care, up to three quarters of all symptoms reported are thought not to be attributable to organic disease. About $25 \%$ of general practice patients have clinically relevant MUS (e.g., Fink et al., 2007; Körber et al., 2011) and $8-10 \%$ of primary care patients have a history of multiple, distressing MUS (e.g., Kroenke et al., 1997). Symptom burden in individuals with MUS seems to be continuously distributed, ranging from non-consulting people with minimal disability (Watson and Pennebaker, 1989) to those with numerous, chronic, severely disabling symptoms (e.g., Jasper et al., 2012).

The economic burden is considerable. In the USA, the annual medical cost of MUS was previously estimated at $\$ 256$ billion (Barsky et al., 2005), while in the UK they are said to account for approximately $10 \%$ of the National Health Service Budget (Bermingham et al., 2010). Up to 42 million work days are lost to MUS in the UK each year (Bermingham et al., 2010), with the associated loss of productivity being estimated at $\$ 19,000$ (US) per patient over 10 years ago (Hiller et al., 2003).

The disease model clearly struggles to accommodate MUS. It is not clear what these conditions should be called (e.g., Creed et al., 2010) or how they should be classified (e.g., Kroenke et al., 2007).Various terms have been used apart from MUS, including "psychosomatic symptoms", "functional symptoms", "subjective health complaints", "somatization", "somatic symptom distress", and "bodily distress". However, there is little agreement on which is most appropriate (Creed et al., 2010; Kroenke et al., 2007) or on the level of description and analysis needed (i.e. as symptoms, syndrome, disorder, or disease). Within general medicine, particular clusters of MUS are often termed functional somatic syndromes, a category that includes irritable bowel syndrome, chronic fatigue syndrome, fibromyalgia and numerous other specialty-specific conditions (Brown, 2007). In psychiatry, particular constellations of MUS are classified as somatoform disorders in the International Classification of Diseases (ICD-10; WHO, 1992), a practice that was mirrored in the Diagnostic and Statistical Manual (DSM-IV-TR; APA, 2000) until its most recent revision when the term "somatic symptom disorder" was coined (DSM-5; APA, 2013). For the somatoform disorders, the emphasis is on symptoms, with diagnoses like somatization disorder (which pertains to individuals with multiple MUS) implying that sufferers have a general tendency to experience MUS that encompasses all bodily systems. This is also true of other systems for classifying patients with multiple MUS (e.g., Fink and Schröder, 2010; Kroenke et al., 1997; Rief and Hiller, 1999), developed in response to concerns about the sensitivity and specificity of the ICD-10 and DSM-IV criteria.

There has been much debate about the overlap between (and within) the functional somatic syndromes and somatoform disorders (e.g., Henningsen et al., 2007; Wessely et al., 1999; Wessely 
and White, 2004; Witthöft et al., 2016), and about the nosological categorisation of the functional syndromes and somatoform disorders as either diseases or (mental) disorders (Geniats, 2015; Jana et al., 2012). In addition, the blurred distinction between MUS and non-MUS has contributed to the recent removal of the somatoform disorders from DSM 5 (APA, 2013) and their replacement with a new Somatic Symptom and Related Disorders category. The centerpiece of this new category, somatic symptom disorder, incorporates all patients with chronic, distressing and/or disabling somatic symptoms who are also exhibiting positive psychological features (e.g., symptom preoccupation, excessive health worry, maladaptive illness behavior), irrespective of whether organic disease has been found. As such, somatic symptom disorder excludes less severe cases of MUS/somatoform disorders compared to DSMIV (Claassen-van Dessel et al., 2016), whist encompassing patients with functional somatic syndromes or documented organic disease where the associated psychological features are also present.

By emphasizing the positive psychological features in response to bodily symptoms, somatic symptom disorder resolves some of the issues regarding the classification of physical symptoms but not others, leading some to propose a return to qualifying diagnoses by whether the somatic symptoms in question can be explained by a biomedical condition (see Rief and Martin, 2014; for a discussion). This illustrates a tension that is likely to remain until the disease model is complemented by a framework that explicitly addresses how consciously perceived symptoms (medically unexplained and otherwise) come about, and when and how they relate (or not) to bodily dysfunction.

\subsection{Somatosensory amplification and misattribution}

Probably the most influential account of MUS has been the somatosensory amplification model (Barsky and Wyshak, 1990). The amplification model assumes that MUS result from stressrelated physiological arousal in threat-sensitive persons, whose illness concerns lead them to misattribute normal sensations to disease causes (e.g., Barsky and Wyshak, 1990; Kolk et al., 2003). Physiological arousal also prompts the individual to focus attention on their body (attentional bias or 'interoceptive hypervigilance'), lowering the threshold for perceiving somatic sensations while priming disease attributions (Barsky and Wyshak, 1990). Misinterpreting the sensations as threatening then causes a further increase in arousal, creating a vicious cycle. The core principles of physiological arousal, hypervigilance and misattribution arguably constitute the modal model of MUS, which is shared by a family of clinical models explaining MUS, hypochondriasis, hyperventilation syndrome and panic disorder. These principles are displayed in Fig. 1.

Variations and elaborations on these themes abound. For example, there is some disagreement among the models as to the necessity of altered physiological arousal: in some models it is assumed that arousal is elevated compared to the normal state of the body, while in other models it is assumed that arousal can be within the normal range but perception is increased because of hypervigilance to it. Exemplars of this family of models are described in Table 1.

The assumptions shared by these models are central to cognitive-behavioral treatments for these complaints, because it is generally thought that there is good evidence for the modal model. We will briefly discuss this evidence.

\subsubsection{Peripheral arousal and stress-related physiology}

The popularity of the amplification model relies in part on the observation that physical symptom reports are commonly comorbid with symptoms of anxiety and depression (Wessely et al., 1999; Kroenke, 2003) and consistently associated $(r=0.40-0.50)$ with higher trait negative affectivity (NA; i.e., a pervasive tendency to experience negative affect; Watson and Pennebaker, 1989) and elevated stress levels (Tak and Rosmalen, 2010). Increased symptom reports in primary care have often been interpreted as resulting from elevated autonomic arousal (Kolk et al., 2003; Kirmayer et al., 2004). It is noteworthy, however, that the most extensive laboratory and ambulatory studies have not found significant differences between MUS reporters and healthy controls across a range of peripheral physiological stress or arousal indicators (e.g., heart rate, cardiac autonomic activity, respiration, salivary cortisol; Houtveen et al., 2010; Houtveen and van Doornen, 2007).

In functional somatic syndromes, where the clinical picture is generally more severe, there is ongoing debate about the importance of physiological abnormalities, with a wide range of possible causes for symptoms being cited, including stress- and

The modal model of symptom perception (see Table 1)

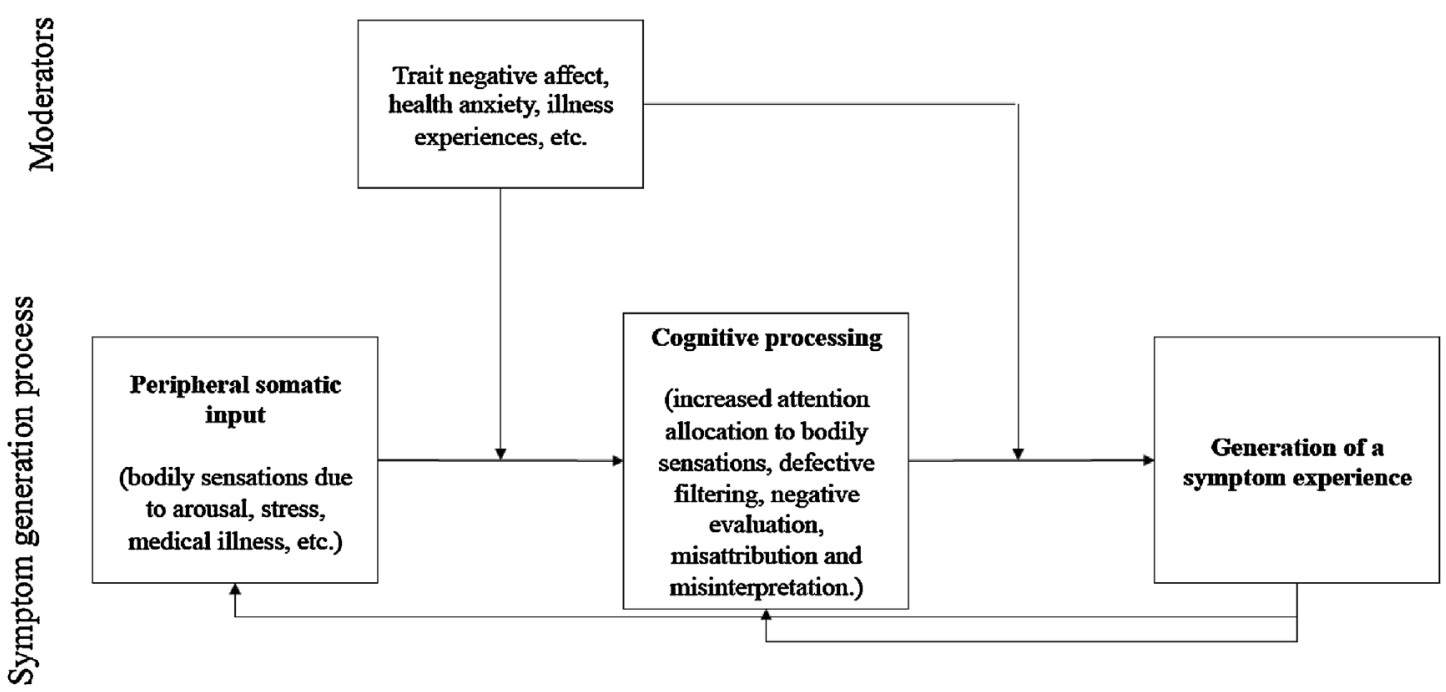

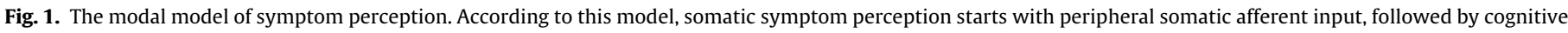
processing that determines the degree of cognitive representation of this input and thereby the strength of conscious symptom perception. 

Scope of the model

Pennebaker (1982);

Watson and Aims to explain the variability in

Pennebaker (1989) somatic symptoms in disease states well as the occurrence of MUS.

Peripheral somatic input
and medically unexplained symptoms and medically unexplained symptoms somatic symptoms in disease sta disorders

Rief and Barsky (2005) _ Aims to explain somatoform disorders and medically unexplained symptom specific focus also on CFS and IBS the core of somatoform disorders and
functional somatic disorders (CFS, FM, functional somatic disorders (CFS, FM, and differences with hypochondriasis may be of low intensity
Periphe;al input is necessary; may res alt from illness processes, from (stress-related) arous or be part of normal bodily sensations.

Attention

Balance between externally and beliefs and personality (e.g. negative including normal bodily sensations. Attention has a focus and direction, but also a content: it can be directed to particular prior hypotheses and specific label for the sensation

Peripheral input is necessary; may result from illness processes, from (stress-related) or be part of normal bodily sensations.

Peripheral input is necessary; may result from physiological diseases processes, from distress, or be part of normal bodily sensations.

Peripheral input is necessary: may result from illness processes, from (stress-related) arousal or be part of normal bodily sensations.

Peripheral input is necessary; may result from illness processes and from (stress-related) arousal.

Lay beliefs and illness representations direct attention to physical symptoms, increasing the chances of them being perceived

Heightened attentional focus on bodily sensations intensifies the experience and leads to the perception of relatively weak sensations that normally remain outside of awareness (amplification)

Attentional processes increase chances for these sensations to be perceived.

Peripheral input is necessary; may result from illness processes, from (stress-related) arousal or be part of normal bodily sensations. Peripheral input is not necessary, chronically activated symptom schemata in an unconscious primary attentional system (PAS) may under some circumstances exceed the sereness threshold and lead to conscious symptom experiences

Several physiological processes are thought to contribute to physical sensations, but they

Genetic vulnerability, personality-related distress, early adverse experiences, life events and HPA-related mechanisms are considered underlying sources of somatic sensations

Experience of bodily stress, resulting from specifiable biological (disease), psychological, interpersonal and/or social factors, is at the core

Physiological processes, as related to alterations in HPA-activity and sustained physiological arousal may underlie somatic sensations, but also media reports. conditioning experiences and chron activated memory schemata may contribute to MUS in the absence of distinct physiological input

External context, selective attention and negative affectivity may influence hightened attention and detection attentional system, concerns may induce elevated vigilance for somatic sensations contributing to chronically active symptom schemat

A failing attentional filter system, to which multiple psychological and biological factors contribute, can cause subthreshold physical sensations to be Attentional biases, possibly resulting from "cognitive sensitization", increase the probability of ensations being perceived.

Attentional mechanisms are not specified, but apparently implied in process of interpretation of stress symptoms as symptoms of a disease

Attentional processes, expectancies and chronically activated somatic memories increase the probability of somatic sensations entering awareness internally directed attention, but also affect) may determine the likelihoo of perceiving bodily sensations,

\section{Attribution and interpretatio}

Beliefs, attributions and interpretations furthe determine how the sensations will be experier ce

The use of a label elicits other meanings and belie structures contributing to (avior

Lay beliefs and illness representations also shape the attribution and interpretation of the sensations.

The sensations are interpreted as threatening and noxious, increasing distress, inducing heightened attention and creating a vicious circle.

Attribution of sensations to illness, illness-related worries and concerns influence distress, illness behavior and help-seeking, which interact with socia and cultural factors. The latter further influence coping with the symptoms.

Meanings and attributions guided by illness beliefs and schemata determine the interpretation of the sensations as specific symptoms Cognitive elaborations guided by illness beliefs and and further determine the interpretation of the symptoms and affect illness behavio

Cognitive, behavioral and emotional processes involved in a vicious circle with biologica mechanisns

Illness attributions raise the threat value of the sensations, feeding back to attentional processes, causing behavioral avoidance and leading to escalating circles, often further reinforced by
insufficient guidance and reassurance by health care insufficient

Attribution and interpretation of bodily stress symptoms as disease may cause anxiety and interpretation as severe disease, and stimulating emotional distress and loss of functioning Catastrophic interpretations and misattributions, importantly driven by neuroticism, amplify somatic sensations, which in turn inspire avoidance behavior and illness interpretations. Inadequate response of health care workers may further fuel the impact of attentional and cognitive processes, including worry, on symptom perception and interpretations. 
disease-related autonomic, endocrine and immune responses. It is important to note in this context that a simple causal model may be too simplistic, and that a distinction should be made between predisposing, precipitating and perpetuating factors. A specific physiological dysfunction eliciting symptoms in an initial stage (e.g. inflammation, infection) may be followed by processes that serve to maintain symptoms, such as stress-related physiology related to, for example, ongoing concerns. In the latter case, however, reliable associations should still be found between symptoms and physiological parameters if symptoms reflect physiological dysfunction.

Meta-analytic and systematic review studies typically reveal a mixed picture. If relationships with physiological abnormalities are found at all, the associations are inconsistent, generally small, and the direction of causality between functional somatic syndromes and the dysfunction remains unclear, mostly leading to the conclusion that there is little convincing evidence for the causal role of a particular physiological dysfunction. This picture applies to autonomic function as indicated by heart rate variability: a metaanalysis by Tak et al. (2009) found no significant difference between patients with functional somatic disorders and healthy controls after controlling for publication bias. Another review found no differences between patients with functional somatic syndromes and healthy controls in half of the studies, and some evidence of reduced cardiac vagal activity in another half, depending also on the type of functional syndrome (Tak and Rosmalen, 2010). A systematic review of Van Cauwenbergh et al. (2014) suggested a reduced cardiac response to a head-up tilt test in chronic fatigue patients in 7 of 8 studies. Taking the cortisol response to indicate hypothalamic-pituitary-adrenal (HPA) axis functioning, Tak et al. (2011) only found evidence for lower cortisol levels in chronic fatigue patients and in females with fibromyalgia, but not in irritable bowel syndrome. A review by Powell et al. (2013) could not establish hypocortisolism in chronic fatigue patients, but found evidence for an attenuation of the diurnal variability of the cortisol response. A similar picture arises from review studies on the role of inflammatory, infectious, or autoimmune dysfunction in functional somatic syndromes: few differences are found, and if so, they mostly pertain to different parameters (see Borchers and Gershwin, 2015; Üçeyler et al., 2011; Blundell et al., 2015; Ishihara et al., 2013; Schwille-Kiuntke et al., 2015). Importantly, whenever a dysfunction is observed, few studies test whether the abnormalities actually cause or mediate the symptoms in question.

\subsubsection{Interoceptive hypervigilance, thresholds and awareness}

Some versions of the amplification model give relatively more weight to hypervigilance and lowered perceptual thresholds for normal physiological arousal. Self-report studies indeed show that individuals with MUS report a tendency to scan the body for signs of illness (e.g., Gendolla et al., 2005; Rief et al., 1998). However, objective measures of attention to health-related stimuli have yielded less consistent findings. For symbolic material (e.g., illness words), some studies have found increased interference on the emotional Stroop task in patients with MUS (e.g., Afzal et al., 2006; Lim and Kim, 2005; Witthöft et al., 2006). These effects may be attributable to increased avoidance of health-threat rather than engagement with it (De Ruiter and Brosschot, 1994), however, or stimulus negativity more generally (Posserud et al., 2009). Studies using the dot-probe and exogenous cueing paradigms have not found evidence of attentional bias in MUS patients (Chapman and Martin, 2011; Hou et al., 2008; Martin and Alexeeva, 2010; Martin and Chapman, 2010; van der Veek et al., 2014; Witthöft et al., 2006).

Studies comparing fibromyalgia patients and healthy controls found no difference in their ability to detect innocuous tactile stimuli (Vandenbroucke et al., 2014; Van Damme et al., 2014), and/or observed that only NA predicted daily symptom reports (Mussgay et al., 1999; Schaefer et al., 2012). Other studies investigating atten- tional processing of bodily sensations themselves provide some evidence for a relationship between attention to the body and symptom reporting, although also implicate avoidance of bodily sensations (Brown et al., 2007; Brown et al., 2010). Interestingly, Katzer et al. (2012) found that lower tactile perceptual thresholds were associated with fewer symptoms in patients with somatoform disorders on the Somatic Signal Detection Task (SSDT). Other studies found that both somatoform disorders (Katzer et al., 2012) and symptom reporting more generally (Brown et al., 2010, 2012; Katzer et al., 2011) were associated with a tendency to report sensory experiences on the SSDT regardless of whether stimuli were actually presented (i.e. 'false alarms'), seemingly contradicting the prediction of improved accuracy. Similarly, Van den Bergh and colleagues found significantly lower correspondence between induced respiratory changes and self-reported breathlessness in a $\mathrm{CO}_{2}$ inhalation paradigm for non-clinical MUS reporters (Bogaerts et al., 2008) and MUS patients (Bogaerts et al., 2010b).

In sum, individuals with MUS consistently report a tendency to scan their bodies for signs of illness, but studies measuring actual attentional deployment towards body- or illness-related stimuli fail to provide convincing evidence for an attentional bias towards these stimuli. Although the available evidence remains too limited for a firm conclusion, most evidence points to a lower correspondence between physiological changes and symptom reports in these individuals.

\subsubsection{Misattribution and interpretation bias}

The modal model assumes that patients with MUS and related conditions show: (i) a tendency to (mis)interpret benign bodily sensations in a negative manner, that is, as overly intense, noxious, and potentially life-threatening (Nakao and Barsky, 2007); and (ii) a tendency to attribute somatic sensations to somatic disease, rather than psychological or neutral/external causes ("somatic attribution bias"). Evidence in line with the (mis)interpretation assumption has been documented for most MUS-related conditions, such as chronic pain, somatoform disorders, fatigue, health anxiety and hypochondriasis (Goedendorp et al., 2013; Marcus et al., 2007; Rief et al., 1998; Rief and Broadbent 2007; Rief and Martin, 2014). These beliefs correlate with various ratings indicating the aversive quality of induced or existent bodily sensations, such as (pain) threshold and unpleasantness. Such beliefs are also reflected in behavioral evidence of a correlation between MUS, health anxiety, hypochondriasis and the automatic negative evaluation of both illness-related pictures (Jasper and Witthöft, 2013), illness words (Schreiber et al., 2014), and aversive tactile stimuli (Witthöft et al., 2012), although this effect was not found in patients with noncardiac chest pain (Schroeder et al., 2014). It is not clear whether such findings point to a cause or consequence of MUS. One recent longitudinal population-based study found that catastrophic misinterpretations of bodily sensations at baseline were a significant predictor of hypochondriacal concerns and fear of bodily sensations 18 months later, but not of physical symptom reports as assessed by a symptom checklist (Woud et al., 2016). Apparently, in this study catastrophic misinterpretations contributed to later cognitive and emotional responses to MUS, but not to the occurrence of MUS themselves. Obviously, replications are needed to confirm this conclusion.

Studies focusing on the attribution style of patients with MUS suggested a dominance of somatic symptom attributions (e.g., Craig et al., 1993; Robbins and Kirmayer, 1991), but recent evidence indicates that they are more complex than this (Hiller et al., 2010) and that somatic attributions do not predict the course of MUS (Douzenis and Seretis, 2013), implying that they are unlikely to play a causal role in the development and maintenance of symptoms. 


\subsubsection{Conclusion}

Although the amplification model is intuitively appealing and remains clinically popular, the available evidence does not provide convincing support for the notion that MUS result from dysregulated peripheral (stress) physiology, hypervigilance for bodily sensations, heightened interoceptive accuracy, or misinterpretations of bodily sensations. The notable lack of evidence that peripheral physiological abnormalities play a specific and causal role in functional somatic syndromes has contributed to growing interest among researchers in the concept of central sensitization as a potential common ground for functional somatic syndromes (see further; Kim and Chang, 2012; Nijs et al., 2012; Bourke et al., 2015). More generally, theorizing seems to have evolved towards identifying MUS as perceptual (or interoceptive) conditions (e.g., Brown, 2004; Edwards et al., 2012).

\subsection{How different are 'explained' and 'unexplained' symptoms?}

If standard medical practice regards MUS and functional somatic syndromes as unusual phenomena that should be exported to the psychological/psychiatric domain, it is generally assumed that the disease model fares better as an account of the symptoms when dysfunction is actually present. Indeed, the correspondence between symptoms and objective physiological parameters is generally high for acute and localized dysfunction or pain (Price et al., 2001). This correspondence is both moderate and highly variable in many multi-symptomatic and chronic diseases, however. For example, there is a poor correspondence between somatic symptoms and objective disease severity in about $50 \%$ of asthma patients depending on the measure (Janssens et al., 2009). In chronic obstructive pulmonary disease (COPD), large scale studies on several thousands of patients showed large between-person variability in the relationship between objective airflow limitation (FEV1) and self-reported breathlessness, with a modest correlation overall ( $r=0.36$, Agusti et al., 2010; $r=0.28$, Müllerová et al., 2014).

Within cardiology, the observed correlation between selfreported symptoms and objective parameters of heart disease (24-h ambulatory monitoring, trans-telephonic ECGs, data from implanted pacemakers or defibrillators) ranges from near zero (Barsky, 2001) to 0.17 (Sears et al., 2005). Similarly, the likelihood of reported arrhythmia symptoms coinciding with an actual arrhythmia ranges from $17 \%$ to $61.1 \%$. Reports of atrial fibrillation have been found in the absence of tachyarrhythmias in $25 \%$ to $45 \%$ of cases (Atarashi et al., 2008; Strickberger et al., 2005). Furthermore, regardless of their effect on objective physiological functioning, trait negative affect, negative emotions, and/or depression have often been found to predict symptom reports better than objective measures of cardiac or respiratory disease (e.g., Janssens et al., 2009; Sears et al., 2005; Van Oudenhove et al., 2008). Data have also shown that a transient increase in stress levels can alter the perception of symptoms in patients with gastro-esophageal reflux disease, resulting in increased symptom reports (Fass et al., 2008; Wright et al., 2005).

Subjective symptom reports correlating poorly with physiological changes have also been found in diabetes, for which accurate detection of health status is of crucial importance. Frankum and Ogden (2005), for example, found that $43.3 \%$ of patients underestimated their blood glucose and $17.3 \%$ overestimated it. Similarly, Ryan et al. (2002) found that estimation of blood glucose was only $28 \%$ accurate for hypoglycemia and $38 \%$ for euglycemia in a sample of adolescents and young adults. Although there have been studies showing greater correlations $(0.70)$ between estimated and actual blood glucose (Schandry et al., 1996), symptom perception in this context is generally considered inaccurate. Evidence also shows that physicians' assessment of symptoms is more highly cor- related with objective organic parameters (0.52-0.92) than those of patients (0.34-0.70; Turner et al., 2010).

In sum, research with medical populations suggests that correlations between symptom reports and objective disease indicators vary substantially, are often low to moderate, and that emotional factors play a particularly significant role in symptom reporting. In other words, a large proportion of the symptoms presented in the context of a well-defined disease could technically be considered "MUS". This is mostly overlooked, however, as few studies actually measure the within-person correspondence between physiological dysfunction and symptom reports.

\subsection{Interim summary}

The divide of western medical systems into either 'physical' or 'mental' health disciplines is arguably responsible for most controversies regarding MUS, with physical and mental health specialists favoring distinct terms, diagnostic criteria and illness narratives for an overlapping set of complaints. Numerous commentators have criticized the oversimplifying mind-body dualism that is inherent to this approach (Rief and Martin, 2014). In addition, a brief excursion into "medically explained diseases" casts doubt on the logic of a clear differentiation between symptoms that do and do not have a physiological explanation. Evidently, there is some continuity in the mechanisms underlying all symptom reporting, whether an 'organic' condition is present or not. Since the seminal monograph of Pennebaker on the psychology of physical symptoms (Pennebaker, 1982), and extensive elaborations in later models (see Cioffi, 1991; Leventhal and Leventhal, 1993; Leventhal et al., 1998), the role of psychological factors in symptom reporting and health care use has been clearly documented. These models typically describe how factors such as beliefs, attributions, emotional states and attention modulate the relationship between physiological dysfunction and symptom reports, but rarely question the basic assumption of the disease model. Moreover, they provide little insight into how consciously perceived symptoms come about, and when or how they relate (or not) to bodily dysfunction. There is a clear need for a symptom perception model that complements the disease model by explaining both "explained" and "unexplained" symptoms, without having to rely on the concept of peripheral physiological change in all cases. We attempt to provide such a framework below.

\section{A new perspective}

\subsection{Aims and central tenets}

In this section we describe a comprehensive model of symptom perception that integrates research and theory on MUS and functional disorders with that on symptom and body perception more generally. Our goal is to describe the mechanisms underlying the conscious experience of somatic symptoms, and thereby the conditions that govern how and when those symptoms correspond with physiological dysfunction. We argue that MUS reflect a perceptual system that is continually generating, testing and refining hypotheses about the causes of sensory inputs, and which is vulnerable to mistaken inferences and false percepts under certain conditions. We suggest that MUS can be regarded as somatovisceral illusions, comparable to visual illusions in casting light on fundamental aspects of perception (also Norman et al., 2014). We claim that this process of automatic and unconscious hypothesis testing applies as much to "veridical" symptom perception (where symptoms correspond closely with physiological dysfunction), as to biased symptom perception (where symptom reports seem only partly consistent with physiological data), and MUS (where no rela- 
tionship with physiological data is found at all). The central tenets of our account can be summarised as follows:

1. Somatic symptoms are conscious percepts that result from a constructive process, in which the brain interprets information from the body in the light of predictions (broadly speaking, expectations) given past experience; this process is moderated by the relative precision afforded to the predictions and the prediction errors;

2. The relationship between parameters of bodily dysfunction and self-reported symptoms is highly variable both between and within individuals over time, depending on interactions between characteristics of the physiological input, the (historical) person and the context; key factors in this respect are those governing the individual's interoceptive sensitivity/acuity and the implicit categorization criteria used to decide whether a sensation is a symptom;

3. The relationship between parameters of bodily dysfunction and self-reported symptoms varies dimensionally. Although MUS are at one end of this continuum, they are functionally comparable both to biased symptom reports of identifiable physiological dysfunction and symptoms experienced in the context of welldescribed diseases;

4. The very process of enquiring about the presence of somatic percepts influences how we experience our body and thereby the symptoms we report.

Our approach is fundamentally different from traditional symptom perception accounts, which assume that “...the perception of physical symptoms is generally preceded by peripheral, physiological changes" (Kolk et al., 2003, p. 2344); see Table 1). It builds on previous accounts of MUS (the Integrative Cognitive Model, ICM; Brown, 2004, 2006, 2013; Brown and Reuber, 2016) and functional neurological and motor symptoms ("conversion symptoms"; Edwards et al., 2012), which assume that peripheral physiological input is neither necessary nor sufficient for symptoms to be experienced. By this view, top-down processes not only influence how, but also whether we experience symptoms. As in the ICM, we regard MUS as distortions in awareness brought about by the over-activation of symptom representations in memory, with various top-down factors serving to maintain this; we move beyond the ICM by integrating our approach more explicitly with existing work on body perception and interoception, and with accounts of the neurobiological substrates of these processes. We also place more emphasis on affective processing, and address certain limitations of the ICM in relation to the role of attention in symptom development and maintenance. Our account follows Edwards et al. (2012) in adopting a predictive coding perspective to help elucidate the mechanisms of MUS. However, Edwards et al. (2012) focuses specifically on functional motor and sensory symptoms (e.g., anaesthesia, movement disorders, sensory loss), and excludes functional symptoms involving autonomic dysfunction and/or arousal, functional syndromes and other somatization problems (Edwards et al., 2012 p. 3496). The latter are the main focus of our paper, in the context of symptom perception more generally. We limit ourselves to processes that help explain how the conscious experience of bodily symptoms comes about, although we acknowledge that symptom perception occurs in an interpersonal and social context that evidently shapes how symptoms are labelled and reported. For the sake of brevity, these social processes are only indirectly taken into account in our model.

We start by describing the basic processes underlying perception of the internal state of the body.

\subsection{Interoception as inference}

Although a continuous, fluctuating array of stimuli impinges on receptors inside our body, most interoceptive information is used by local and subcortical regulation systems and is not amenable to conscious perception. From the limited amount of sensory information that afferent systems can process, the brain could theoretically create an infinite number of patterns of experiences. The task of the brain is to group input into those patterns that are most useful, reducing computational load by ignoring inputs that are unlikely to have adaptive value. Since Helmholtz (1860), numerous theorists (e.g., Gregory, 1980; Friston, 2005; Kveraga et al., 2007; Clark, 2013) have argued that the brain achieves this through an inferential process, involving the creation of probabilistic models about the causes of current inputs to the system, based on prior knowledge. These assumptions have recently been elaborated for interoceptive and affective information processing and their interaction with external perception (Barrett and Bar, 2009; Edwards et al., 2012; Seth, 2013; Barrett and Simmons, 2015). Chronic pain has recently been conceptualized using a predictive coding perspective, as has the modulation of pain perception by placebo and nocebo expectations (see Büchel et al., 2014; Hechler et al., 2016; Wiech, 2016). Our account builds on these approaches.

\subsubsection{Prediction, prediction error and precision}

In predictive coding models, learned knowledge about the world is conceptualized as a set of neural representations or 'priors', which capture the statistical regularities of brain activity. These are represented as probability distributions that describe an expected range of values for a given input and their associated likelihood (Fig. 2). Different prior distributions match sensory inputs ("observation" in Fig. 2) to varying degrees, resulting in prediction errors (i.e., the portion of input not predicted by the prior, or the difference in mean between prior and "observed" distributions).

Every combination of prior and observed distributions constitutes a model of the causes of the actual stimulation, each with a different range of probabilities (posterior distribution).

These generative models initially capture the gist of the stimulus array (Kveraga et al., 2007), and prediction errors are then used to further refine them. A fundamental "motivation" of the system is to minimize prediction error (Friston, 2005). This process can be accomplished by updating the prior to account for unpredicted stimulation (perceptual inference; broadly speaking: changing "expectations"), by generating information that fits the prior through action (active inference), or by changing how input is sampled by the brain (Barrett and Simmons, 2015). The system that accomplishes this is hierarchically organized, such that lower levels of the hierarchy represent the basic properties of the sensory input, with complexity, abstraction and spatio-temporal scale increasing as one proceeds through the hierarchy. There is a continuous, bi-directional flow of information through this hierarchy, such that each level receives predictions from, and feeds back prediction errors to, the levels above. In a continuous interplay of these processes, bottom-up information (prediction errors) is dependent on top-down influences (predictions), which themselves are influenced by previous prediction errors depending on their precision. It also means that dysfunctional predictions will have consequences for predictions errors, and vice versa (see below).

Across a number of experiences, predictions and prediction errors may acquire associated "confidence", represented by the variance around the mean of the distribution (i.e., they have different precisions). A precise prior corresponds to a strong prediction, allowing for perceptual decisions with a high level of confidence. We use the term confidence in a statistical sense only, since these perceptual decisions rarely reach the level of awareness for them 

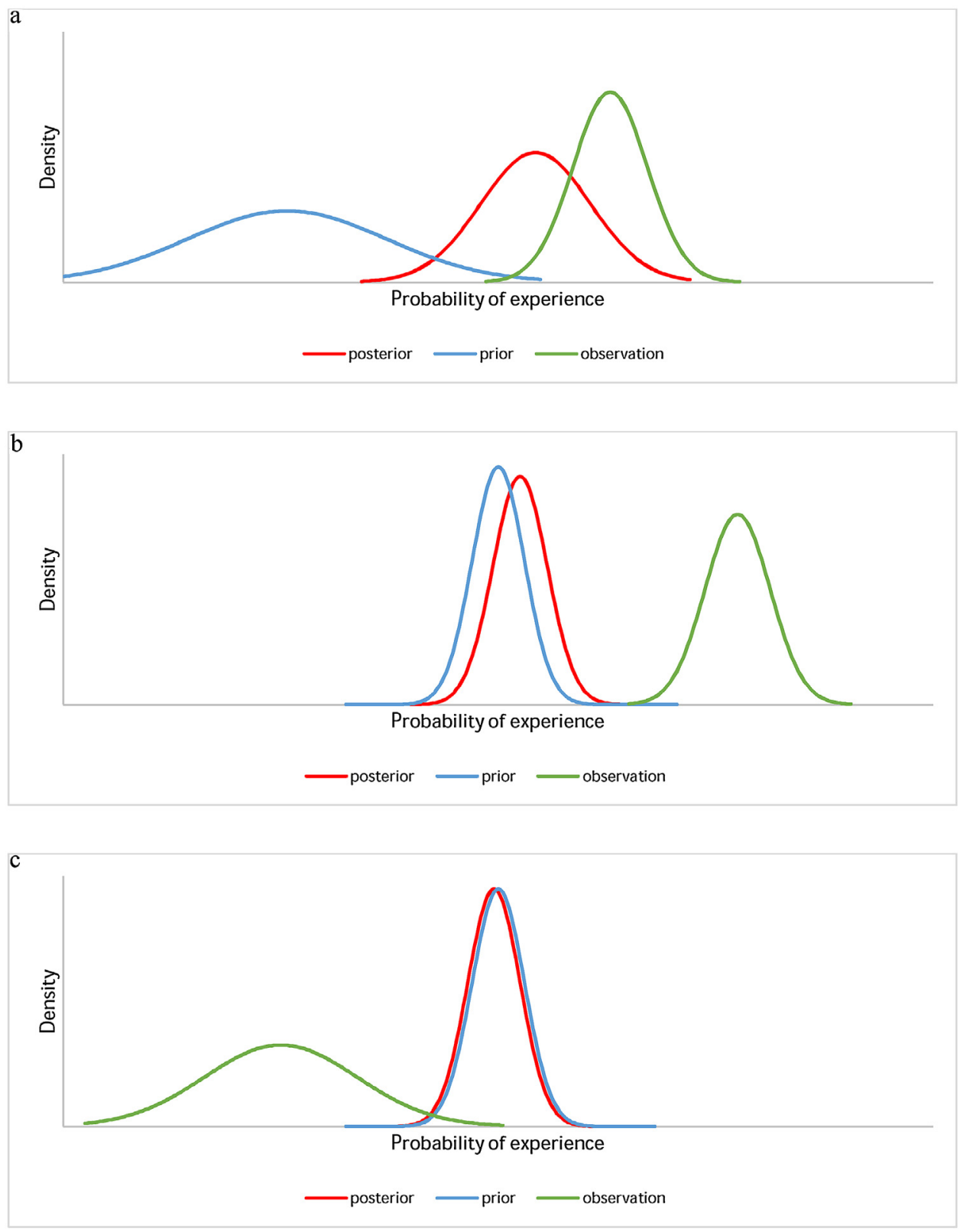

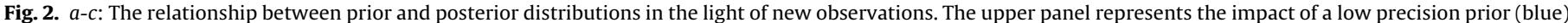

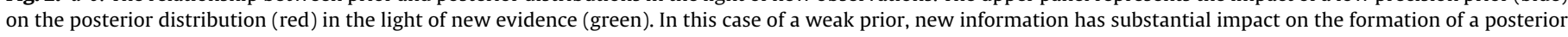

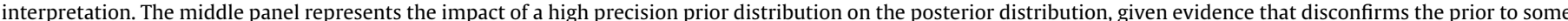

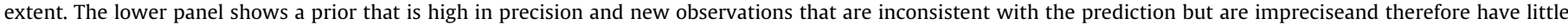

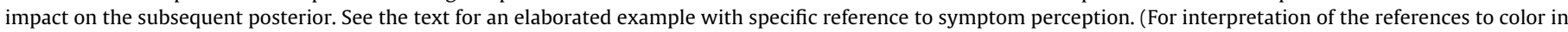
this figure legend, the reader is referred to the web version of this article.)

to manifest as meta-cognitive certainty; instead, the subject simply perceives what the system has concluded is (or is not) there.

Consider the case of a newly developed condition such as asthma, when a patient starts to encounter interoceptive sensations that may or may not represent an asthma symptom. In Fig. 2a, relatively few prior observations are available, meaning that the perceptual system is less certain whether a sensation(e.g., feeling of tightness in the chest) is a relevant indicator of the person's health status. This is represented in Fig. 2a as a relatively broad, flat prior distribution, that is, one with relatively low precision with a central tendency that is located at a low probability (central tendency on the left hand side of the $\mathrm{x}$ axis), indicating that the diagnostic value of this sensation for asthma is low. Comparatively precise new observations (grey line) have a considerable impact on such a vague prior and shift the initial hypothesis to be closer to the new evidence (the posterior; black line). As more observations are made (e.g., over the course of a year), the system learns that the sensation is indeed a valid indicator of asthma, resulting in a narrower, denser (i.e., more precise) prior distribution that shifted to the right as the estimated probability of the sensation being an asthma symptom increased (dashed line in Fig. 2b). The larger amount of past evidence by this point means that the same amount of new information (i.e., new encounters with the sensation; grey line in $2 \mathrm{~b}$ ) has less of an impact on the inference process than before, meaning that the posterior distribution (black line in $2 \mathrm{~b}$ ) remains close to the prior. Fig. $2 \mathrm{c}$ illustrates a case where, in contrast to the first two examples, the probability of a sensation indicating asthma on the basis of prior experiences is high, while the somatic sensation 
(observation) is less precise. Here there is even less of an impact of the new observation, resulting in minimal change from the prior distribution and stronger editing of the incoming information. The result is an experience (posterior) that reflects the prior rather than the observation, with pre-existing expectations being maintained.

In precise distributions there is a greater likelihood that the expected/actual value falls close to the mean, resulting in a larger influence on the generative model. Typically, the model with the lowest overall prediction error is that with the optimal balance between precision and accuracy (i.e., the smallest difference between the means of the predicted and prediction error distributions). An important aspect of the perceptual process is how the system determines whether the prediction is an adequate account of the input. In order to do this, it must be able to estimate the likelihood of any residual prediction error being random noise, or whether it could be reduced further by updating the prediction. The system does so by developing context-dependent expectations about the likely precision of its inputs, and it compensates for these by adjusting the weight placed on them in the perceptual process (Hohwy, 2012). Visual prediction errors are higher in the dark, for example, and relatively more likely to be a product of noise than signal than when it is light. In contrast, the system learns that errors arising in the light are likely to be meaningful (i.e., an unexpected stimulus), and should have more of an influence on perception.

The process of attributing weights to the prediction errors to optimize perception (precision optimization) is thought to be an important factor determining the dominant generative model and therefore conscious percepts (Feldman and Friston, 2010; Hohwy, 2012). Typically, priors have less influence and are more subject to revision when processing involves units expecting precise information from the sensorium (e.g., in the light). Conversely, priors have more of an influence on perception, and are more resistant to updating, when noisy, imprecise sensory input is expected (e.g., in the dark). One consequence of this is that mistakes can be made when unexpected-but-meaningful signals arise when inputs are predicted to be imprecise, biasing perception towards a precise but inaccurate prior. Thus, we may mistake Joe for Fred in the dark if there is a strong expectation that Fred will arrive first. In the account below we develop the idea that MUS involve a similar perceptual error.

\subsubsection{Neurobiological considerations}

Vagus nerve afferents are a major source of interoceptive information, relaying sensory information from nearly every visceral and somatic system to the brain through the nucleus tractus solitarius (NTS) and ascending projections to brainstem, limbic and cortical structures (Berthoud and Neuhuber, 2000). Several brain structures and circuits are critical in constructing and representing a conscious state of the body (e.g., Craig, 2002, 2009) forming an interoceptive nervous system (Harshaw, 2015). For example, the anterior insular cortex is generally considered to play a central role in constructing a multimodal representation of the internal state of the body, integrating hormonal, immunological, metabolic, thermal, autonomic, visceromotor, proprioceptive, exteroceptive, motivational and cognitive sources of information (Craig, 2009; Critchley and Harrison, 2013). The anterior insular cortex is also considered to play a critical role as a source of visceromotor predictions and in matching prediction errors with predictions (Seth, 2013; Seth et al., 2011). Through close connections with the anterior cingulate cortex, these multi-modal representations also involve affective-motivational components and associated approach-avoidance tendencies, consistent with the close connection between interoceptive inference about bodily states and feelings and emotions (Seth, 2013; Zaki et al., 2012). Other structures involved in processing the affective value of interoceptive stimuli are the orbitofrontal (Barrett and Bar, 2009) and ventrome- dial prefrontal cortex, which, together with parts of the cingulate cortex, are thought to constitute a stimulus valuation network (Harshaw, 2015) that engages behavioral control systems when local physiological regulation fails (e.g., gasping for air and opening the window when breathless).

A predictive coding perspective assumes that precision is represented by the action of specific cells that tune the synaptic gain (i.e., post-synaptic responsiveness) of cells encoding predictions and prediction errors (Friston, 2008; Barrett and Simmons, 2015). As there is a constant interplay between priors and prediction errors at multiple hierarchical levels, a predictive coding model surpasses simple and unidirectional conceptions of "top-down" and "bottomup" processes, as well as the notion that specific functions are localized to particular brain regions. Consistent with this, recent neurobiological models have emphasized continuous interactions between counter-flowing streams of information at multiple hierarchical levels. Barrett and Simmons' (2015) Embodied Predictive Interoceptive Coding model describes an integrated neural network that serves both homeostatic and allostatic control functions as well as interoception. It emphasizes more widespread corticocortical connectivity across hierarchically organized lamina (cortical columns) forming granular, agranular and (intermediate) dysgranular cortices. These cortices consist of anatomically different cells acting as prediction, prediction error and precision neurons. Agranular visceromotor cortices comprising mid-cingulate, anterior cingulate cortex, posterior ventromedial prefrontal cortex and parts of the anterior insula generate autonomic, hormonal and immunological predictions to adjust the body to anticipated needs. This information is also sent to granular cortices comprising the mid-to-posterior insula, where prediction errors are calculated and sent back to agranular visceromotor regions; here, outputs to the body are modulated and new interoceptive predictions arise. Visceromotor cortices can also modulate the gain of corticothamalic and thalamocortical connections (i.e., attention to interoceptive sensations).

Importantly, agranular visceromotor regions are considered to be relatively insensitive to prediction error signals due to precisionweighting factors and aspects of the cytoarchitecture. For this reason, interoceptive prediction errors are typically small, meaning that interoceptive perception (the posterior model) is largely dominated by prior expectations. As Barrett and Simmons (2015, p. 424) put it: "interoceptive perception is largely a construction of beliefs that are kept in check by the actual state of the body (rather than vice versa)". Another important feature is that agranular visceromotor cortices are a central hub sending efferent copies of predictions to multiple sensory systems across the brain, and thus subserve "a multisensory representation of the world from the perspective of someone with a body" (Barrett and Simmons (2015, p. 424)). This architecture contributes to embodiment of perception, cognition and emotion, and to bi-directional penetrance (i.e., cross-fertilisation) of interoceptive and exteroceptive information (Harshaw, 2015; for examples). These neurobiological findings place important constraints on theories of symptom perception, which is evidently highly sensitive to prior expectations and contextual cues.

\subsubsection{Interoception, expectations and the sense of self}

Conceptualising interoception as inference blurs the distinction between perceptions and beliefs or expectations. Consistent with this, neurobiological findings show that largely the same brain areas are activated regardless of whether symptoms are produced using expectancy manipulations or elicited by peripheral stimulation. For example, an fMRI study of patients with disabling self-reported electrosensitivity exposed to sham mobile phone radiation found activation in the same brain regions (anterior cingulate cortex; left and right anterior insular cortex) as that 
produced by actual nociceptive stimulation (heat pain; Landgrebe et al., 2008). Similarly, Derbyshire et al. (2004) found that hypnotic suggestions for pain activated the same brain areas as a thermal pain stimulus, including anterior cingulate cortex, anterior insular cortex and somatosensory cortex (S2). In placebo analgesia to experimental pain, activations also emerge in similar brain areas to those involved in processing the sensory and emotional/affective components of pain (Enck et al., 2008).

Interoceptive representations are thought to be central to the sense of self, the experience of body ownership and the feeling of being "present" in the world (Damasio, 2010; Seth, 2013). When conflict is created between interoceptive and exteroceptive information, the process of minimizing prediction error can give rise to somatosensory disturbances in which the core classes of me/not me are confused. In the Rubber Hand Illusion, for example, a fake hand can be experienced as one's own if it is stroked at the same time and rate as the genuine (but concealed) body part (Botvinick and Cohen, 1998). Interestingly, people with poor interoceptive sensitivity tend to report a stronger illusion (Tsakiris et al., 2011). A more prosaic example is the illusory sense of motion that is often experienced when sitting on a stationary train and observing a neighboring train depart. These and other examples illustrate how perceptual inference can misrepresent the true causes of events in the world, giving rise to unusual yet compelling somatosensory experiences. We suggest that similar processes are operating in MUS and other cases of symptom misperception.

\subsection{Interoceptive inference and symptom perception}

Subjective feeling states and embodied selfhood rely on active inference about a multisensory array of interoceptive and exteroceptive signals (Clark, 2013; Seth, 2013; Barrett and Simmons, 2015). Accordingly, the feeling of being healthy may be considered an inference of the experiencing self, whereby somatic prediction errors (e.g., normal somatic variations) are accounted for by predictions regarding what constitutes a "normal body condition". This applies even in the context of continuous and varying input from bodily receptors to the brain, as long as those inputs remain within the predicted range. Subjectively, this is likely to be experienced as the relative absence of interoceptive sensations and bodily awareness. By this view, interoceptive sensations only arise in the event of a sufficient increase in prediction error, with the threshold for the required increase (i.e., the error "tolerance") varying across situations and individuals. A crucial reference point for these prediction errors are the innate values for homeostasis (i.e., 'built-in' priors or predictions about viable physiological functioning) that have emerged from biological evolution (Van De Cruys, 2014). When there is deviation from these homeostatic reference values, the resulting interoceptive error signals will often be minimized by automatic physiological regulation mechanisms. If these low-level mechanisms remain unsuccessful, or when prediction errors are strong and persistent, the errors may give rise to interoceptive sensations (i.e., phenomenal percepts pertaining to the body, associated with varying degrees of conscious awareness) and recruit behavioral control systems to reduce prediction error and re-establish homeostasis.

Aside from the statistical regularities of the inputs themselves and the homeostatic reference values, symptom perception depends on the priors that have been acquired over the course of the individual's learning history concerning the potential causes of somatosensory inputs. At higher levels of representation, this includes abstract information about both normative (e.g., temporary dehydration; physical exercise) and non-normative causes (e.g., disease). The latter are broadly equivalent to the symptom schemata, symptom representations and illness representations described in other models (e.g., Leventhal et al., 1992; Pennebaker,
1982; Cioffi, 1991; Brown, 2004). Whether the input is experienced as a symptom or a sensation depends on the nature of the bestfitting model. We propose that symptoms are experienced when the generative model with the lowest overall prediction error represents an interoceptive event with an abnormal (typically disease) cause. In other words, symptoms arise when the brain interprets interoceptive inputs with reference to predictions about the likely cause of those inputs and infers that there is something wrong with the body (in which 'wrongness' can vary from rather vague ["not well"] to quite specific ["cancer"]).

For each set of inputs there are numerous possible interpretations or predictions, with the phenomenology of the experience being jointly determined by the predictions and inputs with the closest match (see Fig. 2). An important implication of this is that there are many different ways of experiencing a set of inputs, which vary according to the parameters of the available predictions. Where the generative model is characterized by a highly accurate prediction, the associated experience will correspond closely with the sensory input (i.e., the correlation between subjective reports and objective physiology will be maximized). Crucially, however, highly accurate predictions may not be part of the optimal model, depending on the precision weights associated with the predictions and the prediction errors (see below). An important implication of this is that distorted (as in symptom under- and over-reporting) or 'false' perceptions (as in MUS) can arise if the weight of the prediction makes it part of the optimal model, despite accuracy (i.e., the match between the prediction and the input) being low.

Building on the work of Edwards et al. (2012), we assume that symptom reports that are decoupled from sensory input - and MUS in the extreme case - arise when, in the presence of predictive cues, excessive precision is afforded to priors predicting the presence of symptoms/disease, rendering those priors the optimal model regardless of whether they are a good match for sensory input. As a result, the individual's experience is distorted in the direction of the priors. How much the experience is related to objective physiology will depend on the extent of the mismatch between the prior and the sensory input and their relative precision weights. In most disease states, highly precise error signals emerge from multiple sources in spatiotemporal proximity (e.g., cough, fatigue, running nose); these will typically combine with contextual cues (e.g., a partner having a cold) to promote precise priors that explain away the prediction errors, leaving the person with a clear illness experience ("I have a cold") that closely corresponds with measurable evidence.

Minor distortions may occur when there is some correspondence between the prior and the input (i.e., the distance between the means of their distributions is relatively small) but the precision of the prior pulls the mean of the posterior distribution towards it; in this case, the experience will be an exaggeration of the input or particular aspects of it (e.g., making it more painful than might be expected given the stimulus). In other cases, the mismatch between the prior and the input may be more substantial, generating a subjectively real but objectively illusory experience (e.g., MUS; also Edwards et al., 2012). Studies demonstrating the acquisition of symptoms through associative learning (e.g., Van den Bergh et al., $1995,1997,2002$ ) suggest this can occur when frequent pairing of a cue with a veridical (i.e., objectively triggered) somatic experience raises the predictive validity of the associated prior, giving it greater perceptual influence in subsequent encounters with the cue. If the precision of the prior becomes sufficiently high, the cue may come to trigger the experience in the absence of the input. These studies, which involved pairing a harmless odor with $\mathrm{CO}_{2}$-enriched air inhalation across repeated breathing trials, found that the conditioning effect was only observed if the odor was negative and only in high NA individuals and MUS patients, suggesting that the effect is dependent on there being a plausible relationship between the cue 
and symptom experience, and a tendency to perceive bodily threat. This is relevant for chronic disease, where different contextual cues may come to promote the underlying generative model as symptoms are experienced over time. As a result, symptom reports may gradually become decoupled from physiology and more dependent on contextual cues (De Peuter et al., 2005; Janssens et al., 2009). Recent research on pain-related placebo and nocebo effects suggests that these processes can arise in the absence of conscious perception (Jensen et al., 2015). Verbal expectancy manipulations, such as those used during hypnotic suggestion and placebo/nocebo paradigms, are also effective ways of creating (or removing) symptom experiences, influencing both neurobiological and peripheral physiological systems at multiple hierarchical levels ranging from cortical to spinal (Atlas and Wager, 2014; Büchel et al., 2014; Enck et al., 2013; Jubb and Bensing, 2013).

In sum, symptom experiences may correspond to varying degrees with peripheral somatic input, depending on the interplay between prediction errors, priors and their relative precisions. Various contextual and individual factors modulate this correspondence, the four main types of which are considered below.

\subsection{Factors influencing symptom perception}

\subsubsection{Varieties of afferent input}

Interoceptive signals can vary along numerous dimensions, such as intensity, quality, location, extent and duration (e.g., compare toothache and fatigue). Although agranular visceromotor regions are considered relatively insensitive to prediction error signals (Barrett and Simmons, 2015; see above), we assume that more intense and localized signals (e.g., a racing heart) will generate more precise prediction errors that are likely to modify and update priors accordingly, resulting in an experienced stimulus. How that sensation is experienced depends on the priors that predict the likely consequences of the input. Thus, a benignly pounding heart might be experienced as a neutral sensation in the context of recent exercise, or as a potential heart attack in the presence of cues suggesting a possible disease cause. In both cases, perceptual detection may be good (i.e., associated with accurate heartbeat detection), but perceptual categorization is inaccurate in the latter.

There is greater scope for highly precise but inaccurate priors to dominate the generative model for somatic stimuli with less precise prediction errors, such as those that are weaker, more systemic and widespread, characterized by poor on/off boundaries and/or when the boundaries with other sensation categories are blurred. Examples of such symptoms include fatigue, inflammation-induced "malaise" and somatic input from stress-related HPA-axis activation. Imprecise prediction errors may also result from various types of interoceptive dysfunction that influence the quality and resolution of the somatic signals that eventually determine conscious symptom perception (Harshaw, 2015; Schulz and Vögele, 2015). For example, there is experimental evidence that both cytokines (Eisenberger et al., 2009; Harrison et al., 2009a,b) and stress/HPA-axis activation influence structural and/or functional characteristics of interoceptive brain areas (Stein et al., 2007; Liston et al., 2009; Gianaros et al., 2007). Similarly, evidence suggests that early life adversity and chronic stress reduce the density and functionality of $\alpha 2$-adrenoceptors in the NTS, which may further compromise sensitive processing of afferent signals from the viscera (Schulz and Vögele, 2015). Genetic factors may also contribute to low signal-to-noise ratios in interoceptive sensitivity (Holliday et al., 2010; Gazouli et al., 2016). Such mechanisms may account for the link between inflammatory and stress-related variables, functional somatic syndromes and increased symptom reports.

In conditions characterized by both imprecise priors and prediction errors, contextual and individual difference variables may have a particularly significant impact on symptom experiences, decou- pling them from physiology. For example, it is possible to induce symptoms in nonclinical and clinical MUS patients simply by presenting them with unpleasant pictures, followed by cues (such as the questions on a symptom scale) promoting attention to particular models of their somatic state (Constantinou et al., 2013; Constantinou et al., 2015).

Decoupled symptoms will reflect the beliefs that are represented by the priors in question, suggesting that differences in beliefs will largely account for the differences in clinical phenomenology. If a belief reflects a particularly precise prior about the presence of a certain experience, it might manifest as a single, debilitating unexplained symptom but low symptom reporting more generally. Where an individual has broader health concerns (i.e., low precision priors) but the prediction errors are also imprecise, a tendency to experience multiple symptoms may result, as in habitual symptom reporting and patients with the historic DSMIV diagnosis of somatization disorder. It is likely that with broader health concerns, individual sensitivities and contextual cues may give more weight to some priors than others, coloring the presentation accordingly. For example, critical incidents pertaining to bowel function may result in greater precision being afforded to gastrointestinal symptoms, whereas prior exposure to viral infection may result in similar emphasis being placed on exhaustion and pain. This would explain how the same set of complaints can end up attracting different diagnoses (e.g., irritable bowel syndrome vs. chronic fatigue syndrome) depending on the medical specialty where they are encountered (Wessely et al., 1999).

\subsubsection{Varieties of attention}

Attentional modulation of visceromotor prediction errors, which influences the balance of precision weights between priors and prediction errors, is thought to be implemented by gain mechanisms in the anterior insula and anterior cingulate cortex (Corbetta and Shulman, 2002) and in corticothalamic connections influencing the thalamic reticular nucleus (Zikopoulos and Barbas, 2006; Barrett and Simmons, 2015). This gain mechanism is attracted to causal regularity in the world, giving a probabilistic advantage to generative models that maximize the signal-to-noise ratio (Hohwy, 2012). When a cue promoting attention is valid (i.e., predictive), it facilitates perception of the corresponding stimuli; when it is invalid, subsequent stimuli will be at a perceptual disadvantage (Hohwy, 2012). As precision weights vary according to individual and contextual factors, these factors will influence the accuracy of somatic perception.

If there are no cues directing attention to the body, minor prediction errors may go unnoticed. In contrast, if priors (e.g., illness-related beliefs or worries) increase the gain on interoceptive error units (i.e., tilt the balance between precision weights of priors and prediction errors towards the latter, thereby increasing 'body-focus'), relatively weak interoceptive stimulation will be represented as stronger and more precise, reflected in more intense or salient conscious percepts. This is a type of self-fulfilling prophecy: an expectation for a strong bottom-up signal increasing the strength of that signal. The increased prediction error will also motivate the system to update its priors in order to account for it. In this context, a model that attributes the inputs to physical illness might be the optimal way of explaining away the interoceptive error, particularly in the presence of relevant cues (e.g., those suggesting a possible health threat). Eventually a self-perpetuating cycle may arise, with illness-related worrying raising the precision of associated priors to the point where a disease model pertains, whether interoceptive stimuli are present or not. In other words, a symptom may begin as the amplification of a weak somatic input but end up as a somatosensory false alarm in which noise is misrepresented as signal. This account unifies the somatosensory amplification and ICM within a common framework, while at 
the same time describing critical mechanisms contributing to the maintenance and chronicity of symptoms over time.

Where somatic signals are expected to be imprecise (due to low intensity/ambiguous inputs, noisy receptor systems etc.) there will be relatively greater weighting of prior models concerning the body. In the absence of precise error feedback to revise those models, they are likely to become increasingly divorced from sensory input over time. This fits with the evidence reviewed above linking MUS with poor interoceptive accuracy, which is likely to result in an expectation of imprecise sensory inputs.

Several contextual and individual factors may influence the gain and thereby symptom experiences. Scanning for signs of illness, for example, is a form of active inference, whereby the system selectively samples sensory inputs with a view to confirming its own predictions (cf. "confirmatory bias"). The precision of the signal being scanned for is relevant here, with the nature of the individual's illness beliefs influencing where the gain is applied. If a relatively precise threat is predicted, for example, then the perception of signals that correspond to that threat will be optimized (making them more likely to become conscious). There will not be a broader increase in gain on other units, and no lowering of interoceptive thresholds more generally, unless the illness predictions are more non-specific. This has clear implications for the choice of stimuli used in studies of attentional bias, which need to be tailored to the particular beliefs of each participant.

It is important to note that in the current model, attention is simply the process by which gain is applied to predictions and prediction error units, increasing or decreasing their relative weight in the perceptual process. Conscious contents, on the other hand, are determined by the generative model with the lowest overall prediction error. By this view, there is a close correspondence between what is "attended to" (i.e., receives gain) and what the individual is conscious of (as gain increases the likelihood of the model being optimal), but the two concepts are nevertheless separable (Hohwy, 2012).

\subsubsection{Gender}

One of the most consistent findings in this area is that women report more, more intense and more frequent symptoms than men, a difference that remains after controlling for specific female gender symptoms (Barsky et al., 2001). MUS, somatoform disorders and functional somatic syndromes are also consistently more prevalent in women than men across clinical and non-clinical settings (e.g., Aamland et al., 2014; Kroenke and Spitzer, 1998; Wessely et al., 1999; Cloninger et al., 1986). A wide variety of potentially related factors might account for this gender difference in symptom reporting, including biological differences in nociception, exposure to early adversity (Edwards et al., 2003), and neuroendocrine stress responses (Bartley and Fillingim, 2016; Doom et al., 2013). Genderrelated differences in symptom appraisal, socialization processes and gender roles, as well as gender biases in research and clinical practices, may also account for some of the effect (Barsky et al., 2001). However, two additional observations are important in this respect: First, in laboratory environments, women are consistently found to be less accurate than men at detecting physiological changes in, for example, heart rate, blood glucose, blood pressure, respiration, and gastrointestinal sensations (Pennebaker and Roberts, 1992; Roberts and Pennebaker, 1994), which may be due to gender-related structural and functional differences in the interoceptive network in the brain (Naliboff et al., 2003; Fairclough and Goodwin, 2007; Harshaw, 2015). Second, this laboratory difference in interoceptive accuracy disappears in natural environments, which may be related to women being more sensitive to contextual cues when determining their internal state (see Pennebaker and Roberts, 1992; Roberts and Pennebaker, 1994; Pennebaker, 1995). An implication is that contextually driven priors are likely to have a greater influence on women's interoception (and therefore symptom perception), while prediction errors resulting from somatic input are more likely to influence men than women. This perspective predicts that this will be more the case in conditions where somatic input is less intense and/or localized, that is, where there is more room for priors to impact symptom perception, which is consistent with gender effects becoming typically evident in the symptom ratings of healthy groups, and in assessments of MUS, somatoform disorders, functional somatic syndromes and organic pathology (Barsky et al., 2001). It also suggests that the gender difference will be more pronounced in somatic disease with a broader range of low intensity symptoms than in acute conditions with a smaller number of intense and localized symptoms, with this difference becoming more pronounced over time as associations between contextual factors and symptom episodes develop.

\subsubsection{Threat and negative affect (NA)}

Elevated physical symptom reporting is consistently associated with trait NA, as well as with anxiety states and affective disturbance, regardless of whether objective disease is present. Importantly, high NA appears to be a vulnerability factor for MUS when it interacts with increased self-focused attention (Gendolla et al., 2005), with previous experiences of somatic events and/or with somatic concerns (Bogaerts et al., 2014; Van den Bergh et al., 1997). As trait anxiety is associated with elevated sensitivity to threat (Hariri, 2009; Yiend, 2010) and compromised inhibitory systems for counter-regulating unpleasantness, it is likely that these mechanisms are involved in the association between high trait NA persons and MUS (Bishop, 2009; Montoya et al., 2005; Tillisch et al., 2011; Van Oudenhove and Aziz, 2013). Recently, the concept of central sensitization has been advanced to capture the idea of hyper-responsivity to various somatosensory stimuli in patients with functional somatic syndromes, characterized by elevated threat and salience detection, and a reduced capacity to down-regulate emotional responses (Bourke et al., 2015; Nijs et al., 2012 for reviews).

Greater activation of affective networks and compromised inhibitory systems may generate augmented and imprecise interoceptive prediction errors (i.e., a greater discrepancy between predicted and actual interoceptive state; Paulus and Stein, 2006), whilst influencing the likelihood of priors being activated that predict the presence of threat, including symptoms in MUS-prone persons. Following Barrett and Bar (2009), we assume that the 'gist' of new stimuli are first captured and used to create initial predictions as a basis for further processing. This process, thought to be governed by the medial orbito-frontal cortex, instigates autonomic and endocrine changes representing predictions about the emotional and motivational significance of the signal, allowing stimuli to be categorized as either aversive, appetitive or neutral, and facilitating object recognition and motivating rapid action (e.g., approach, avoid) where appropriate. It also gives stimuli their hedonic tone (i.e., whether, and to what extent, they are experienced as pleasant or unpleasant; Barrett and Bar, 2009). Stimuli that are characterized as potentially threatening or aversive at an early stage are afforded processing priority (e.g., Robinson et al., 2013) aimed at reducing the threat and thereby minimizing prediction error.

At the point of initial threat categorization, generative models are necessarily approximate: a detailed, contextually relevant generative model that accounts for the prediction errors follows later and evolves over time (Barrett and Bar, 2009; Kveraga et al., 2007). As such, the initial experience may simply be one of an aversive interoceptive experience associated with a non-specific sense that "something is wrong". Evidence suggests that one way of reducing ambiguity about the cause of this experience might be to alter the sampling strategy (Barrett and Simmons, 2015), by reducing 
detailed sensory-perceptual processing of the prediction errors and shifting the focus of representation to a simple categorization about the nature of the threat. Consistent with this, a set of studies requiring participants to categorize respiratory resistances of varying intensity levels (Petersen et al., 2014) found that anxious persons showed poorer discrimination among within-category interoceptive stimuli and fitted stimuli into categorical priors by taking less note of the variability resulting from sensory-discriminative processing (Procrustes effect; Petersen et al., submitted; Petersen et al., 2015a). Anxiety was also related to an increasing lack of differentiation between interoceptive stimuli over time, a generalization process which may serve to disambiguate stimuli in the short term, but lead to higher error feedback over time. In another study, high anxious persons reporting high levels of symptoms in daily life misclassified low respiratory resistances close to the category border into a high category, indicating a liberal criterion for identifying stimuli as symptoms consistent with a "better safe than sorry" strategy (Petersen et al., 2015b). There is also evidence that anxiety during pain processing is associated with diminished perceptual discrimination of pain-related stimuli (Zaman et al., 2015).

Other studies in high trait NA persons with MUS showed that self-reported symptoms became less strongly related to objective indicators of physiological dysfunction (as induced by $\mathrm{CO}_{2}$-inhalation) when the latter was administered in a negative affective context. This effect only appeared when participants were asked to rate "symptoms" (e.g., dyspnea/breathlessness) and not when rating neutrally labelled "sensations" (e.g., breathing intensity), showing that a reduction in sensory-perceptual detail is only applied when contextual cues advance symptom-related priors (Bogaerts et al., 2005, 2008, 2010b). Less detailed sensoryperceptual processing of somatic episodes in SSD patients may also underlie the absence of a peak-end bias when evaluating previous somatic episodes (Bogaerts et al., 2012) and less specific healthrelated autobiographical memories (Walentynowicz et al., 2016).

If detailed sensory-perceptual processing is reduced, interoceptive prediction errors will be imprecise, enabling high-level priors to become potent biasing factors of somatic experiences. There is evidence that patients with MUS hold more precise priors about "a normal body condition" (Rief et al., 1998) and have lower tolerance for uncertainty, leading to more prediction errors and thereby symptoms, especially when questioned about their somatic state. Also chronic somatic concerns may afford more weight to symptom-related priors, reducing detailed sensoryperceptual processing. This allows the prediction errors associated with negative affective states to be construed as somatic symptoms when conditions promote symptom-related priors, a finding that has been observed consistently with high NA persons in a negative affective state (Bogaerts et al., 2010a; Constantinou et al., 2013). Indeed, in a state of chronic uncertainty and stagnated error reduction, it may be adaptive to take an inferential leap on the basis of insufficient data, adopting a model that explains away somatosensory prediction errors and paradoxically reduces the overall level of threat; in other words, it may be better to know that you are ill than to be unsure whether you are (i.e., "better the devil you know"). Whilst disambiguation may serve a short term goal of reduced aversive feedback, however, it sustains a high error rate in the long run; the result is chronic negative affect and further attempts at disambiguation, expressed as chronic worry about the state (Carleton et al., 2014) and the creation of a vicious circle that ultimately leads to chronic MUS and somatoform disorders.

\subsection{Summary}

One of the main advantages of the model described here is that it integrates research and theory from separate literatures those on MUS and those on symptom reporting more generally - within a unifying framework that specifies how symptoms come to be perceived, and the conditions under which objective and subjective health markers diverge. In this new model, the conscious experience of a somatic symptom comes about as a result of the continuous interplay between expectations and evidence where specific factors and conditions specify the relative weight of both sources of information in determining the eventual experience (see Fig. 3).

An advantage of the model is that a categorical boundary between MUS and medically explained symptoms is replaced by a process that allows gradual and context-dependent changes in the relationship between objective health markers and selfreported symptoms, and in which MUS emerge as extreme (but common) instances of this process. It also helps to understand how symptoms that initially were closely linked to objective disease indicators eventually may become decoupled from it, why there is a predominance of women showing MUS, and why threat sensitive individuals are more prone to develop MUS. The model also describes several mechanisms contributing to the maintenance of symptoms and their development into chronic complaints, which are relevant regardless of how much the symptoms correspond to objective disease indicators.

\section{Implications}

We conclude by briefly highlighting some of the implications of the model at different levels.

\subsection{Theoretical implications}

A central premise of this framework is that the brain can only make sense of the world by being sensitive to statistical regularities in its own neural activity. Moreover, how and how well we perceive our internal state is always contextualized, that is, predicated on specific factors within the person and context, meaning that the "truthfulness" of perception is always relative. These aspects have a number of important implications.

First, in order to understand MUS, the main issue may not be whether interoception is accurate or inaccurate, but why and how MUS become valid (i.e., are adaptive) in a pragmatic sense (Petersen et al., 2011). Whilst it is likely that adaptive models of the world often correspond closely with regularities in the stimulation, less accurate or even distorted models may sometimes be more useful if they are efficient (i.e., minimize the amount of time and energy needed to test them) and predict important events well enough (Kruglanski, 1989; Lynn and Barrett, 2014). This highlights the need for studies that test aspects of symptom perception such as the precision of priors and the decision strategies used to classify sensations as noise or signal (i.e., "normal" or "symptom"). A window into confidence in perceptual hypotheses may be offered by intra-individual variance in the perception of the same stimulus, as precision is the inverse of variability (inverse dispersion). The perceiver may or may not be aware of this variance/confidence, however.

Second, contrary to the somatosensory amplification model, and perhaps common clinical wisdom, our account assumes that there is no necessary relationship between interoceptive sensitivity as investigated in studies assessing detection thresholds for interoceptive stimulation and symptom reports of physiological dysfunction. Although sensitivity may have a bearing on whether a sensation reaches awareness, symptom perception and reporting is more a matter of classifying those sensations into categories associated with threat. Context plays a crucial role in this process, as predictions and their associated precision weights differ dramatically from one situation to the next. Counting heart beats in 
A predictive coding approach to symptom perception

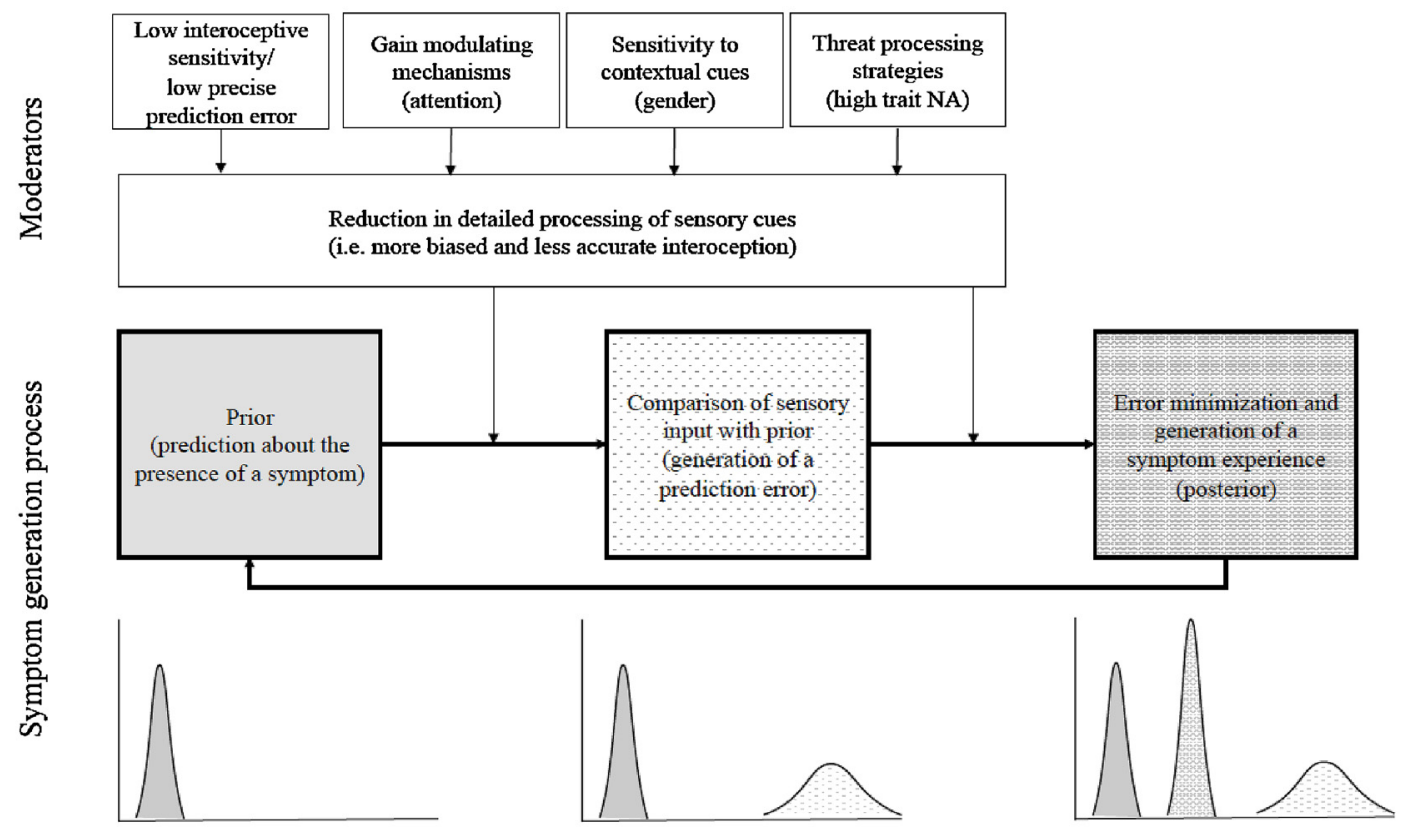

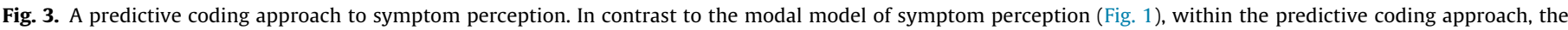

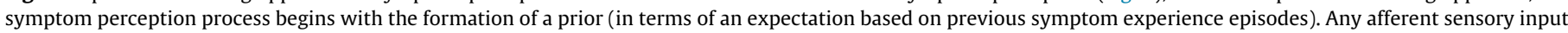

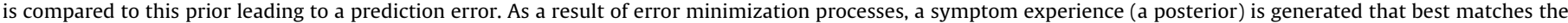
prior and the prediction error. The posterior serves as input determining the prior in a new symptom perception episode.

an experimental context is quite different, for example, from perceiving heart beats whilst walking up the stairs, having just read a newspaper story about the sudden cardiac arrest and death of a celebrity.

Third, moderators or individual risk factors for MUS, such as NA or gender, are not factors influencing a supervising agent (or self) that perceives the internal state, but contribute directly to perception itself. Since interoception is a continuous process in which hypotheses are tested and adapted in multiple trials, moderating traits act as inherent characteristics of the algorithms underlying the perceptual process and not as an external factor influencing perception. Since the precision of prior distributions rather than their central tendency will affect information seeking and thereby model adjustment, a shift in research from means to the precision of distributions is needed.

\subsection{Clinical implications}

\subsubsection{Diagnostic implications}

As the brain creates educated guesses about somatic stimulation based on likelihoods, it is important to test not only the end-product of interoception (e.g., classification of a sensation, estimates of intensity, unpleasantness, location), but also the interoceptive process itself. It could therefore be informative for health care professionals to be able to assess the nature and precision of their patients' priors and the disambiguation and classification strategies they use in relation to interoceptive information. Several benefits may emerge from developing the present perspective into diagnostic strategies. First, if bias in interoception is not regarded as "measurement error", but as the patient's best guess resulting from a specific mental model, this takes away any blame or stigma towards them for being wrong in an absolute sense. Second, the boundaries between normal and pathological cases would become blurred because any symptom report can be disconnected from objective physiological indicators to some extent, depending on the context and history of the person. This would at the same time acknowledge the empirical evidence that MUS vary in a dimensional way and occur in "objective disease" as well as in the absence of it. Third, assessing mediating processes rather than end-products points directly to intervention strategies, consistent with current calls to move away from labels and categorical diagnoses and to focus on transdiagnostic markers and processes (RDoC initiative, Cuthbert and Insel, 2013). We believe that the development of diagnostic tools focusing on intra-individual variability in classifying the same interoceptive stimulus, and on variations in disambiguation and classification strategies as a function of contextual cues, may provide valuable information in this respect.

\subsubsection{Treatment implications}

Following the modal models in this area, the dominant treatment strategies involve the development of various self-help techniques such as (1) reducing physiological arousal (e.g., through relaxation); (2) altering interoceptive (hyper)vigilance; and (3) correcting (catastrophic) misinterpretations of somatic sensations. Studies of psychological treatments for MUS in general show relatively low effect sizes (Kleinstäuber et al., 2011; Van Dessel et al., 2014) and there is much room for improvement. A major weakness the somatosensory amplification and signal filtering models have in common is that they do not flesh out the process by which conscious symptom experiences come about in the first place. In the present model, symptoms emerge in consciousness when the generative model with the lowest overall prediction error and best ratio of complexity and efficiency represents an interoceptive event with an abnormal or disease cause. However, the processes and interoceptive algorithms that lead to that experience are not available for introspection (i.e., are outside awareness), which results in a strong and immediate feeling that one's somatic experience is trustworthy ("sensing is believing"). Since this process is the same whether there is a close correlation with objective disease indicators or not, it fits with the perspective of the patient who experiences no difference between MUS and non-MUS symptoms. 
Breaking the "sensing is believing" cycle and encouraging the formation of new generative models may require specific experiences repeated over time. Interoceptive exposure therapy is probably the most fruitful track to follow but the technique may need further fine-tuning. In its present form it is intended to reduce interoceptive fear, that is, to reduce confidence in the immediate negative outcome of a sensation, which may also implicitly increase tolerance for uncertainty. From the present perspective, however, it is also necessary to reduce confidence in the presence of a sensation/symptom itself (sensing is believing) as well as to increase the perceived heterogeneity of sensation and symptom categories. To reduce implicit confidence that a sensation is a symptom, training people to become more sensitive to differences in interoceptive sensations may be more helpful (Schaefer et al., 2014). This may be achieved by shifting the level of representation under conditions of threat from affective-categorical to sensory-perceptual, thereby reducing the weight of the priors. Collectively these therapeutic strategies might be termed "interoceptive differentiation training".

\subsection{Translating the model into testable hypotheses}

The model points to several obvious and important challenges for research, including the independent assessment of priors, prediction errors and their relative precisions at the neural levels, how they are influenced by contextual variables and individual differences, and how they relate to behavioral processes. A more general implication can be derived from the central assumption in our approach, namely that the brain compresses information into classes that optimize the balance between redundancy and loss of unique information (Chater and Vitányi, 2003; Seger and Peterson, 2013). This categorization process involves implicit inferences about causes and consequences of the stimulation, resulting from the interplay between the distributions of priors and prediction errors. This focus on distributions is different from the traditional view that typically assesses mean values such as the location of a sensation on a magnitude or unpleasantness scale, and tends to consider intra-individual variance as measurement error. In contrast, we suggest that variance is a valuable window into confidence, that is, into the precision of prior and posterior distributions (defining precision as inverse dispersion). Intra-individual variance can pertain to the same stimulus (ratings by one person for one stimulus presented repeatedly to investigate the role of situational factors or dynamic changes over time) or to different stimuli (sensitivity for differences between interoceptive stimuli). Intra-individual variance in processing complexity (i.e., the number and degree of independence of interoceptive dimensions involved in stimulus evaluation) may also provide useful information. For example, pain can be sharp and not dull, and dyspnea can feel more like air hunger than chest tightness. When category complexity and inter-dimensional variance are reduced, categories become more inclusive (more sensations fit the simplified prototype) and misclassification is more likely for symptom categories that are represented in this simplified fashion.

An example of this approach is a recent study in which a set of equidistant respiratory resistances varying in intensity was administered to a group of healthy participants. Subsequently, the lower resistances were artificially grouped into category $A$, and the higher resistances were grouped into category $B$. Inducing these artificial categories caused both assimilation and accentuation effects, that is, the perceived differences between stimuli within categories were suppressed while the differences between categories were accentuated (Petersen et al., 2014). These findings clearly illustrate the role of interoceptive categories as priors.

Such methods may reveal how contextual, state and traitrelated individual difference variables change the processing algorithms underlying symptom perception. Some effects may result from the relative impact of specific (types of) priors, while other effects may result from different sensitivities to detect sensory-perceptual differences, or from implicitly used decision strategies to classify sensations. For example, a recent study showed that threat sensitivity is positively associated with the degree to which interoceptive information processing is condensed by assimilation and accentuation, and with a sense of increased certainty about one's classifications (Petersen et al., 2015b). The curtailment of detailed sensory-perceptual processing and the disambiguation of interoceptive information via assimilation and accentuation (allowing for an inferential leap) could be regarded as a quick and dirty categorization strategy that may be appropriate if resources are low and/or the need for disambiguation (error reduction) is high (e.g., a liberal "better safe than sorry" strategy; see Petersen et al., 2015b; supra).

These are just a few examples of how the present perspective suggests novel research methods for studying interoceptive processing and symptom perception, shedding new light on how symptom experiences relate to objective bodily events.

\section{Summary and conclusions}

The basic assumption underlying the model presented here is that the brain makes sense of the internal state of the body by being sensitive to statistical regularities in its own neural activity. It does this by compressing information into categories in a parsimonious way, optimizing the balance between redundancy and loss of unique information, in order to form mental representations of the bodily state. These representations (generative models) exist at multiple hierarchical levels and are continuously shaped and refined by mapping neural activity representing prior expectations onto incoming afferent activity. This inferential process eventually results in abstract categorical representations reaching awareness, for example, in the form of consciously felt pain, weakness or breathlessness. We propose that consciously experienced symptoms reflect the generative model with the lowest overall prediction error representing an interoceptive event with an abnormal (typically disease) cause. Depending on specific conditions, the percept of the body may be more influenced by prior expectations or by actual inputs (prediction errors).

We conclude by listing what we consider to be the major strengths of the model. First, the dynamic interplay between priors and prediction errors at multiple hierarchical levels results in a dimensional variation of the relationship between subjectively experienced physical symptoms and objective physical dysfunction. This is consistent with a wealth of evidence showing that the strength of this relationship varies enormously in organic disease; importantly, it accommodates MUS within the same framework, placing them at the extreme end of this dimension. Future work may consider how other seemingly anomalous phenomena, such as placebo, nocebo, phantom limb pain, and hypnotic effects could be understood using a similar framework (Büchel et al., 2014). One consequence of this approach is that medicine, in order to become truly patient-focused, needs to go beyond the biopsychosocial perspective and embrace a symptom perception model. Second, the relationship between the experience of physical symptoms and objective physical dysfunction is always contextualized, that is, influenced by specific factors within the historical person, the context, and their interaction. This means that the relationship between symptoms and physiological dysfunction may vary substantially both within-person and within-situation. Third, the model de-emphasizes the importance of "accuracy" in symptom perception and promotes the importance of understanding its validity and utility in a pragmatic sense. This shift in emphasis has important theoretical and clinical consequences. Fourth, clin- 
ical intervention should more explicitly target those inferential processes leading to the phenomenal experience of symptoms as "really there". Fifth, and most importantly, a predictive coding framework may suggest novel behavioral paradigms as well as new measurement parameters for testing critical predictions. By extending these approaches to include neurobiological paradigms, we hope to provide an antidote to a narrow disease model and the unhelpful separation of psyche and soma.

\section{Conflict of interest}

None of the authors has a conflict of interest.

\section{Authors contribution}

All authors contributed in a significant way during all phases of writing this manuscript. All authors have read and approved the final manuscript.

\section{Acknowlegements}

Omer Van den Bergh was funded by grant OT-10-027 and by a Center for Excellence grant "Generalization in Health and Psychopathology" from the Research Council of the University of Leuven. Sibylle Petersen was funded by grant AFR PDR 2013-2 of the Fonds National de la Recherche Luxembourg.

The authors are grateful for careful reading and constructive comments during earlier stages of this manuscript to the following persons: Elena Constantinou, Thomas Janssens, Ellen Poliakoff, Lukas Van Oudenhove, Sander Van de Cruys, Andreas von Leupoldt, Johan Wagemans, and Marta Walentynowicz.

\section{References}

Üçeyler, N., Häuser, W., Sommer, C., 2011. Systematic review with meta-analysis: cytokines in fibromyalgia syndrome. BMC Musculoskelet. Disord. 12, 245, http://dx.doi.org/10.1186/1471-2474-12-245.

American Psychiatric Association, 2000. Diagnostic and Statistical Manual of Mental Disorders: Text Revision (DSM-IV-TR). American Psychiatric Pub.

American Psychiatric Association, 2013. Diagnostic and Statistical Manual of Mental Disorders: (DSM-5 ${ }^{\circledast}$ ). American Psychiatric Pub http://dx.doi.org/10. 1176/appi.books.9780890425596.

Aamland, A., Malterud, K., Werner, E.L., 2014. Patients with persistent medically unexplained physical symptoms: a descriptive study from Norwegian genera practice. BMC Fam. Pract. 15 (1), 1.

Afzal, M., Potokar, J.P., Probert, C.S., Munafò, M.R., 2006. Selective processing of gastrointestinal symptom-related stimuli in irritable bowel syndrome. Psychosom. Med. 68, 758-761, http://dx.doi.org/10.1097/01.psy.0000232270. 78071.28.

Agusti, A., Calverley, P.M., Celli, B., Coxson, H.O., Edwards, L.D., Lomas, D.A., Tal-Singer, R., 2010. Characterisation of COPD heterogeneity in the ECLIPSE cohort. Respir. Res. $11(1), 1-14$.

Atarashi, H., Ogawa, S., Inoue, H., 2008. Relationship between subjective symptoms and trans-telephonic ECG findings in patients with symptomatic paroxysmal atrial fibrillation and flutter. J. Cardiol. 52, 102-110, http://dx.doi.org/10.1016/ j.jjcc.2008.06.006.

Atlas, L.Y., Wager, T.D., 2014. A meta-analysis of brain mechanisms of placebo analgesia: consistent findings and unanswered questions. In: Bendetti, F., Enck, P., Frisaldi, E., Schedlowski, M. (Eds.), Placebo. Springer Berlin Heidelberg Berlin, pp. 37-69.

Büchel, C., Geuter, S., Sprenger, C., Eippert, F., 2014. Placebo analgesia: a predictive coding perspective. Neuron 81, 1223-1239, http://dx.doi.org/10.1016/j. neuron.2014.02.042.

Barrett, L.F., Bar, M., 2009. See it with feeling: affective predictions during object perception. Phil. Trans. R. Soc. B : Biol. Sci. 364, 1325-1334, http://dx.doi.org/ 10.1098/rstb.2008.0312.

Barrett, L.F., Simmons, W.K., 2015. Interoceptive predictions in the brain. Nat. Rev. Neurosci. 16, 1-11, http://dx.doi.org/10.1038/nrn3950.

Barsky, A.J., Wyshak, G., 1990. Hypochondriasis and somatosensory amplification. Brit. J. Psychiatry 157, 404-409.

Barsky, A.J., Peekna, H.M., Borus, J.F., 2001. Somatic symptom reporting in women and men. J. Gen. Intern. Med. 16 (4), 266-275.

Barsky, A.J., Orav, E.J., Bates, D.W., 2005. Somatization increases medical utilization and costs independent of psychiatric and medical comorbidity. Arch. Gen. Psychiatry 62, 903-910, http://dx.doi.org/10.1001/archpsyc.62.8.903.
Barsky, A.J., 2001. Palpitations, arrhythmias, and awareness of cardiac activity. Ann. Intern. Med. 134, 832-837, http://dx.doi.org/10.7326/0003-4819-134-9 Part_2-200105011-00006.

Bermingham, S.L., Cohen, A., Hague, J., Parsonage, M., 2010. The cost of somatisation among the working-age population in England for the year 2008-2009. Mental Health Family Med. 7, 71.

Berthoud, H.R., Neuhuber, W.L., 2000. Functional and chemical anatomy of the afferent vagal system. Auton. Neurosci. 85 (1), 1-17.

Bishop, S.J., 2009. Trait anxiety and impoverished prefrontal control of attention. Nat. Neurosci. 12, 92-98, http://dx.doi.org/10.1038/nn.2242.

Blundell, S., Ray, K.K., Buckland, M., White, P.D., 2015. Chronic fatigue syndrome and circulating cytokines: a systematic review. Brain Behav. Immun. 50, 186-195.

Bogaerts, K., Notebaert, K., Van Diest, I., Devriese, S., De Peuter, S., Van den Bergh O., 2005. Accuracy of respiratory symptom perception in different affective contexts. J. Psychosom. Res. 58, 537-543, http://dx.doi.org/10.1016/j. jpsychores.2004.12.005.

Bogaerts, K., Millen, A., Li, W., De Peuter, S., Van Diest, I., Vlemincx, E., Fannes, S., Van den Bergh, O., 2008. High symptom reporters are less interoceptively accurate in a symptom-related context. J. Psychosom. Res. 65, 417-424, http:// dx.doi.org/10.1016/i.jpsychores.2008.03.019.

Bogaerts, K., Janssens, T., De Peuter, S., Van Diest, I., Van den Bergh, O., 2010a. Negative affective pictures can elicit physical symptoms in high habitual symptom reporters. Psychol. Health 25, 685-698, http://dx.doi.org/10.1080/ 08870440902814639.

Bogaerts, K., Van Eylen, L., Li, W., Bresseleers, J., Van Diest, I., De Peuter, S., Stans, L. Decramer, M., Van den Bergh, O., 2010b. Distorted symptom perception in patients with medically unexplained symptoms. J. Abnorm. Psychol. 119, 226, http://dx.doi.org/10.1037/a0017780.

Bogaerts, K., Wan, L., Van Diest, I., Stans, L., Decramer, M., Van den Bergh, O., 2012. Peak-end memory bias in laboratory-induced dyspnea: a comparison of patients with medically unexplained symptoms and healthy controls. Psychosom. Med. 74, 974-981, http://dx.doi.org/10.1097/PSY. 0b013e318273099c.

Bogaerts, K., Rayen, L., Lavrysen, A., Van Diest, I., Janssens, T., Schruers, K., Van den Bergh, O., 2014. Unraveling the relationship between trait negative affectivity and habitual symptom reporting. PLoS One 10, http://dx.doi.org/10.1371/ journal.pone.0115748.

Borchers, A.T., Gershwin, M.E., 2015. Fibromyalgia: a critical and comprehensive review. Clin. Rev. Allergy Immunol. 49 (2), 100-151.

Botvinick, M., Cohen, J., 1998. Rubber hands 'feel' touch that eyes see. Nature 391, 756, http://dx.doi.org/10.1038/35784.

Bourke, J.H., Langford, R.M., White, P.D., 2015. The common link between functional somatic syndromes may be central sensitization. J. Psychosom. Res. 78, 228-236.

Brown, R.J., Reuber, M., 2016. Towards an integrative theory of psychogenic non-epileptic seizures (PNES). Clin. Psychol. Rev. 47, 155-170, http://dx.doi. org/10.1016/j.cpr.2016.06.003.

Brown, R.I., Poliakoff, E., Kirkman, M.A., 2007. Somatoform dissociation and somatosensory amplification are differentially associated with attention to the tactile modality following exposure to body-related stimuli. J. Psychosom. Res. 62, 159-165, http://dx.doi.org/10.1016/j.jpsychores.2006.08.008.

Brown, R.J., Danquah, A.N., Miles, E., Holmes, E., Poliakoff, E., 2010. Attention to the body in nonclinical somatoform dissociation depends on emotional state. J Psychosom. Res. 69, 249-257, http://dx.doi.org/10.1016/i.jpsychores.2010.04. 010.

Brown, R.J., Skehan, D., Chapman, A., Perry, E.P., McKenzie, K.J., Lloyd, D.M., Babbs, C., Paine, P., Poliakoff, E., 2012. Physical symptom reporting is associated with a tendency to experience somatosensory distortion. Psychosom. Med. 74, 648-655, http://dx.doi.org/10.1097/PSY.0b013e3182595358.

Brown, R.I., 2004. Psychological mechanisms of medically unexplained symptoms: an integrative conceptual model. Psychol. Bull. 130, 793, http://dx.doi.org/10. 1037/0033-2909.130.5.793.

Brown, R.J., 2006. Medically unexplained symptoms: a new model. Psychiatry 5, 43-47, http://dx.doi.org/10.1383/psyt.2006.5.2.43.

Brown, R.J., 2007. Introduction to the special issue on medically unexplained symptoms: background and future directions. Clin. Psychol. Rev. 27, 769-780, http://dx.doi.org/10.1016/j.cpr.2007.07.003.

Brown, R.J., 2013. Dissociation and somatoform disorders. In: Kennedy, F., Kennerley, H., Pearson, D. (Eds.), Cognitive Behavioural Approaches to the Understanding and Treatment of Dissociation. Routledge, London, pp. 133-147.

Carleton, R.N., Duranceau, S., Freeston, M.H., Boelen, P.A., McCabe, R.E., Antony, M.M., 2014. But it might be a heart attack: intolerance of uncertainty and panic disorder symptoms. J. Anxiety Disord. 28, 463-470, http://dx.doi.org/10.1016/ j.janxdis.2014.04.006.

Chapman, S., Martin, M., 2011. Attention to pain words in irritable bowel syndrome: increased orienting and speeded engagement. Br. J. Health Psychol. 16, 47-60, http://dx.doi.org/10.1348/135910710x505887.

Chater, N., Vitányi, P., 2003. Simplicity: a unifying principle in cognitive science? Trends Cogn. Sci. 7, 19-22, http://dx.doi.org/10.1016/S1364-6613(02)00005-0.

Cioffi, D., 1991. Beyond attentional strategies: a cognitive-perceptual model of somatic interpretation. Psychol. Bull. 109, 25-41, http://dx.doi.org/10.1037// 0033-2909.109.1.25.

Claassen-van Dessel, N., van der Wouden, J.C., Dekker, J., van der Horst, H.E., 2016 Clinical value of DSM IV and DSM 5 criteria for diagnosing the most prevalent somatoform disorders in patients with medically unexplained physical 
symptoms (MUPS). J. Psychosom. Res. 82, 4-10, http://dx.doi.org/10.1016/j. jpsychores.2016.01.004.

Clark, A., 2013. Whatever next? Predictive brains, situated agents, and the future of cognitive science. Behav. Brain Sci. 36, 181-204, http://dx.doi.org/10.1017/ S0140525x12000477.

Cloninger, C.R., Martin, R.L., Guze, S.B., Clayton, P.I., 1986. A prospective follow-up and family study of somatization in men and women. Am. J. Psychiatry 143 (7), 873-878.

Constantinou, E., Bogaerts, K., Van Diest, I., Van den Bergh, O., 2013. Inducing symptoms in high symptom reporters via emotional pictures: the interactive effects of valence and arousal. J. Psychosom. Res. 74, 191-196, http://dx.doi. org/10.1016/i.jpsychores.2012.12.015.

Constantinou, E., Van Den Houte, M., Bogaerts, K., Van Diest, I., Van den Bergh, O., 2015. Can words heal? Using labeling to reduce the effects of unpleasant cues on symptom reporting. Front. Psychol.: Emot. Sci. 5, 807, http://dx.doi.org/10. 3389/fpsyg.2014.00807.

Corbetta, M., Shulman, G.L., 2002. Control of goal-directed and stimulus-driven attention in the brain. Nat. Rev. Neurosci. 3 (3), 201-215.

Craig, T.K., Boardman, A.P., Mills, K., Daly-Jones, O., Drake, H., 1993. The South London Somatisation Study I: Longitudinal course and the influence of early life experiences. Br. J. Psychiatry 163, 579-588, http://dx.doi.org/10.1192/bjp. 163.5.579.

Craig, A.D., 2002. How do you feel? Interoception: the sense of the physiologica condition of the body. Nat. Rev. Neurosci. 3, 655-666, http://dx.doi.org/10. 1038/nrn894.

Craig, A.D., 2009. How do you feel now? The anterior insula and human awareness Nat. Rev. Neuroscience 10, 59-70, http://dx.doi.org/10.1038/nrn2555.

Creed, F., Guthrie, E., Fink, P., Henningsen, P., Rief, W., Sharpe, M., White, P., 2010. Is there a better term than medically unexplained symptoms? J. Psychosom. Res. 68, 5-8, http://dx.doi.org/10.1016/i.jpsychores.2009.09.004.

Critchley, H.D., Harrison, N.A., 2013. Visceral influences on brain and behavior. Neuron 77, 624-638, http://dx.doi.org/10.1016/j.neuron.2013.02.008.

Cuthbert, B.N., Insel, T.R., 2013. Toward the future of psychiatric diagnosis: the seven pillars of RDoC. BMC Med. 11, 126.

Damasio, A., 2010. Self Comes to Mind. Pantheon, New York.

De Peuter, S., Van Diest, I., Lemaigre, V., Li, W., Verleden, G., Demedts, M., Van den Bergh, O., 2005. Can subjective asthma symptoms be learned? Psychosom. Med. 67, 454-461.

De Ruiter, C., Brosschot, J.F., 1994. The emotional Stroop interference effect in anxiety: attentional bias or cognitive avoidance. Behav. Res. Ther. 32, 315-319, http://dx.doi.org/10.1016/0005-7967(94)90128-7.

Deary, V., Chalder, T., Sharpe, M., 2007. The cognitive behavioural model of medically unexplained symptoms: a theoretical and empirical review. Clin. Psychol. Rev. 27, 781-797, http://dx.doi.org/10.1016/j.cpr.2007.07.002.

Derbyshire, S.W., Whalley, M.G., Stenger, V.A., Oakley, D.A., 2004. Cerebral activation during hypnotically induced and imagined pain. Neuroimage 23, 392-401, http://dx.doi.org/10.1016/j.neuroimage.2004.04.033.

Doom, J.R., Cicchetti, D., Rogosch, F.A., Dackis, M.N., 2013. Child maltreatment and gender interactions as predictors of differential neuroendocrine profiles. Psychoneuroendocrinology 38 (8), 1442-1454.

Douzenis, A., Seretis, D., 2013. Descriptive and predictive validity of somatic attributions in patients with somatoform disorders: a systematic review of quantitative research. J. Psychosom. Res. 75, 199-210, http://dx.doi.org/10. 1016/j.jpsychores.2013.05.005.

Edwards, V.I., Holden, G.W., Felitti, V.I., Anda, R.F., 2003. Relationship between multiple forms of childhood maltreatment and adult mental health in community respondents: results from the adverse childhood experiences study. Am. J. Psychiatry 160 (8), 1453-1460.

Edwards, M.J., Adams, R.A., Brown, H., Pareés, I., Friston, K.J., 2012. A bayesian account of 'hysteria'. Brain 135, 3495-3512, http://dx.doi.org/10.1093/brain aws 129.

Eisenberger, N.I., Inagaki, T.K., Rameson, L.T., Mashal, N.M., Irwin, M.R., 2009. An fMRI study of cytokine-induced depressed mood and social pain: the role of sex differences. Neuroimage 47, 881-890 http://dx.doi.org/10.1016/i. neuroimage.2009.04.040.

Enck, P., Benedetti, F., Schedlowski, M., 2008. New insights into the placebo and nocebo responses. Neuron 59, 195-206, http://dx.doi.org/10.1016/j.neuron. 2008.06.030.

Enck, P., Bingel, U., Schedlowski, M., Rief, W., 2013. The placebo response in medicine: minimize, maximize or personalize? Nat. Rev. Drug Discov. 12 , 191-204, http://dx.doi.org/10.1038/nrd3923.

Fairclough, S.H., Goodwin, L., 2007. The effect of psychological stress and relaxation on interoceptive accuracy: implications for symptom perception. I. Psychosom. Res. 62 (3), 289-295.

Fass, R., Naliboff, B.D., Fass, S.S., Peleg, N., Wendel, C., Malagon, I.B., Mayer, E.A., 2008. The effect of auditory stress on perception of intraesophageal acid in patients with gastroesophageal reflux disease. Gastroenterology 134, 696-705, http://dx.doi.org/10.1053/j.gastro.2007.12.010.

Feldman, H., Friston, K.J., 2010. Attention, uncertainty, and free-energy. Front. Hum. Neurosci. 4, 215, http://dx.doi.org/10.3389/fnhum.2010.00215.

Fink, P., Toft, T., Hansen, M.S., Ørnbøl, E., Olesen, F., 2007. Symptoms and syndromes of bodily distress: an exploratory study of 978 internal medical, neurological, and primary care patients. Psychosom. Med. 69, 30-39, http://dx. doi.org/10.1016/j.jpsychores.2010.02.004.

Frankum, S., Ogden, J., 2005. Estimation of blood glucose levels by people with diabetes: a cross-sectional study. Br. J. Gen. Pract. 55, 944-948.
Friston, K., 2005. A theory of cortical responses. Phil. Trans. R. Soc. B : Biol. Sci. 360, 815-836, http://dx.doi.org/10.1098/rstb.2005.1622.

Friston, K., 2008. Hierarchical models in the brain. PLoS Comput. Biol. 4, http://dx. doi.org/10.1371/journal.pcbi.1000211.

Gazouli, M., Wouters, M.M., Kapur-Pojskić, L., Bengtson, M.B., Friedman, E., Nikčević, G., Niesler, B., 2016. Lessons learned - resolving the enigma of genetic factors in IBS. Nat. Rev. Gastroenterol. Hepatol. 13, 77-87, http://dx. doi.org/10.1038/nrgastro.2015.206.

Gendolla, G.H., Abele, A.E., Andrei, A., Spurk, D., Richter, M., 2005. Negative mood, self-focused attention, and the experience of physical symptoms: the joint impact hypothesis. Emotion 5, 131, http://dx.doi.org/10.1037/1528-3542.5.2. 131.

Geniats, T.G., 2015. Redifining the chronic fatigue syndrome. Ann. Intern. Med. 162 653-654, http://dx.doi.org/10.7326/M15-0357.

Gianaros, P.I., Horenstein, J.A., Cohen, S., Matthews, K.A., Brown, S.M., Flory, J.D. Hariri, A.R., 2007. Perigenual anterior cingulate morphology covaries with perceived social standing. Soc. Cogn. Affect. Neurosci. 2, 161-173 http://dx.doi. org/10.1093/scan/nsm013.

Goedendorp, M.M., van der Werf, S.P., Bleijenberg, G., Tummers, M., Knoop, H., 2013. Does neuropsychological test performance predict outcome of cognitive behavior therapy for Chronic Fatigue Syndrome and what is the role of underperformance? J. Psychosom. Res. 75, 242-248, http://dx.doi.org/10.1016/ j.jpsychores.2013.07.011.

Gregory, R.L., 1980. Perceptions as hypotheses. Phil. Trans. R. Soc. B : Biol. Sci. 290, 181-197.

Hariri, A.R., 2009. The neurobiology of individual differences in complex behavioral traits. Annu. Rev. Neurosci. 32, 225-247, http://dx.doi.org/10.1146/annurev. neuro.051508.135335.

Harrison, N.A., Brydon, L., Walker, C., Gray, M.A., Steptoe, A., Critchley, H.D., 2009a. Inflammation causes mood changes through alterations in subgenual cingulate activity and mesolimbic connectivity. Biol. Psychiatry 66 (5), 407-414.

Harrison, N.A., Brydon, L., Walker, C., Gray, M.A., Steptoe, A., Dolan, R.J., Critchley H.D., 2009b. Neural origins of human sickness in interoceptive responses to inflammation. Biol. Psychiatry 66 (5), 415-422 http://dx.doi.org/10.1016/j. biopsych.2009.03.007.

Harshaw, C., 2015. Interoceptive dysfunction: toward an integrated framework for understanding somatic and affective disturbance in depression. Psychol. Bull. 141 (2), 311

Hechler, T., Endres, D., Thorwart, A., 2016. Why harmless sensations might hurt in individuals with chronic pain: about heightened prediction and perception of pain in the mind. Front. Psychol. 7, http://dx.doi.org/10.3389/fpsyg.2016. 01638.

Helmholtz, H.V., 1860. Theorie der luftschwingungen in Röhren mit offenen enden. Journal für die Reine und Angewandte Mathematik 57, 1-72.

Henningsen, P., Zipfel, S., Herzog, W., 2007. Management of functional somatic syndromes. Lancet 369, 946-955, http://dx.doi.org/10.1016/S01406736(07)60159-7.

Hiller, W., Fichter, M.M., Rief, W., 2003. A controlled treatment study of somatoform disorders including analysis of healthcare utilization and cost-effectiveness. J. Psychosom. Res. 54, 369-380, http://dx.doi.org/10.1016 S0022-3999(02)00397-5.

Hiller, W., Rief, W., Brähler, E., 2006. Somatization in the population: from mild bodily misperceptions to disabling symptoms. Soc. Psychiatry Psychiatr. Epidemiol. 41, 704-712, http://dx.doi.org/10.1007/s00127-006-0082-y.

Hiller, W., Cebulla, M., Korn, H.J., Leibbrand, R., Röers, B., Nilges, P., 2010. Causal symptom attributions in somatoform disorder and chronic pain. J. Psychosom. Res. 68, 9-19, http://dx.doi.org/10.1016/j.jpsychores.2009.06.011.

Hohwy, J., 2012. Attention and conscious perception in the hypothesis testing brain. Front. Psychol. 3, 96, http://dx.doi.org/10.3389/fpsyg.2012.00096.

Holliday, K.L., Macfarlane, G.J., Nicholl, B.I., Creed, F., Thomson, W., McBeth, J., 2010. Genetic variation in neuroendocrine genes associates with somatic symptoms in the general population: results from the EPIFUND study. J. Psychosom. Res. 68, 469-474, http://dx.doi.org/10.1016/j.jpsychores.2010.01.024.

Hou, R., Moss-Morris, R., Bradley, B.P., Peveler, R., Mogg, K., 2008. Attentional bias towards health-threat information in chronic fatigue syndrome. J. Psychosom. Res. 65, 47-50, http://dx.doi.org/10.1016/j.jpsychores.2008.03.008.

Houtveen, J.H., van Doornen, L.J., 2007. Medically unexplained symptoms and between-group differences in 24-h ambulatory recording of stress physiology. Biol. Psychol. 76, 239-249, http://dx.doi.org/10.1016/j.biopsycho.2007.08.005.

Houtveen, J.H., Hamaker, E.L., Van Doornen, L.J., 2010. Using multilevel path analysis in analyzing 24-h ambulatory physiological recordings applied to medically unexplained symptoms. Psychophysiology 47, 570-578, http://dx. doi.org/10.1111/j.1469-8986.2009.00951.x.

Ishihara, S., Tada, Y., Fukuba, N., Oka, A., Kusunoki, R., Mishima, Y., Kinoshita, Y 2013. Pathogenesis of irritable bowel syndrome-review regarding associated infection and immune activation. Digestion 87, 204-211, http://dx.doi.org/10 $1159 / 000350054$

Jana, A.K., Praharaj, S.K., Mazumdar, J., 2012. Current debates over nosology of somatoform disorders. Ind. Psychiatry J. 21, 4-10, http://dx.doi.org/10.4103/ 0972-6748.110939.

Janssens, T., Verleden, G., De Peuter, S., Van Diest, I., Van den Bergh, O., 2009. Inaccurate perception of asthma symptoms: a cognitive-affective framework and implications for asthma treatment. Clin. Psychol. Rev. 29, 317-327, http: // dx.doi.org/10.1016/j.cpr.2009.02.006. 
Jasper, F., Witthöft, M., 2013. Automatic evaluative processes in health anxiety and their relations to emotion regulation. Cognit. Ther. Res. 37, 521-533, http://dx. doi.org/10.1007/s10608-012-9484-1.

Jasper, F., Hiller, W., Rist, F., Bailer, J., Witthöft, M., 2012. Somatic symptom reporting has a dimensional latent structure: results from taxometric analyses. J. Abnorm. Psychol. 121, 725-738, http://dx.doi.org/10.1037/a0028407.

Jensen, K., Kirsch, I., Odmalm, S., Kaptchuk, T.J., Ingvar, M., 2015. Classical conditioning of analgesic and hyperalgesic pain responses without conscious awareness. Proc. Natl. Acad. Sci. 112, 7863-7867, http://dx.doi.org/10.1073/ pnas.1504567112.

Jubb, J., Bensing, J.M., 2013. The sweetest pill to swallow: how patient neurobiology can be harnessed to maximise placebo effects. Neurosci. Biobehav. Rev. 37, 2709-2720, http://dx.doi.org/10.1016/j.neubiorev.2013.09.006.

Körber, S., Frieser, D., Steinbrecher, N., Hiller, W., 2011. Classification characteristics of the Patient Health Questionnaire-15 for screening somatoform disorders in a primary care setting. J. Psychosom. Res. 71, 142-147, http://dx.doi.org/10.1016/j.jpsychores.2011.01.006.

Katzer, A., Hiller, W., Oberfeld, D., Witthöft, M., 2011. Tactile perceptual processes and their relationship to medically unexplained symptoms and health anxiety. J. Psychosom. Res. 71, 335-341, http://dx.doi.org/10.1016/j.jpsychores.2011. 03.009 .

Katzer, A., Oberfeld, D., Hiller, W., Gerlach, A.L., Witthöft, M., 2012. Tactile perceptual processes and their relationship to somatoform disorders. J. Abnorm. Psychol. 121, 530, http://dx.doi.org/10.1037/a0026536.

Kim, S.E., Chang, L., 2012. Overlap between functional GI disorders and other functional syndromes: what are the underlying mechanisms? Neurogastroenterol. Motil. 24, 895-913, http://dx.doi.org/10.1111/i.13652982.2012.01993.x.

Kirmayer, L.J., Taillefer, S., 1997. Somatoform disorder. In: Turner, S.M., Hersen, M. (Eds.), Adult Psychopathology and Diagnosis. , 2nd ed. John Wiley \& Sons, New York, pp. 333-383

Kirmayer, L.J., Groleau, D., Looper, K.J., Dao, M.D., 2004. Explaining medically unexplained symptoms. Can. J. Psychiatry 49, 663-672, http://dx.doi.org/10. 1177/070674370404901003.

Kleinstäuber, M., Witthöft, M., Hiller, W., 2011. Efficacy of short-term psychotherapy for multiple medically unexplained physical symptoms: a meta-analysis. Clin. Psychol. Rev. 31, 146-160, http://dx.doi.org/10.1016/j.cpr. 2010.09.001.

Kolk, A.M., Hanewald, G.J., Schagen, S., van Wijk, C.M.G., 2003. A symptom perception approach to common physical symptoms. Soc. Sci. Med. 57, 2343-2354, http://dx.doi.org/10.1016/S0277-9536(02)00451-3.

Kroenke, K., Spitzer, R.L., 1998. Gender differences in the reporting of physical and somatoform symptoms. Psychosom. Med. 60 (2), 150-155.

Kroenke, K., Spitzer, R.L., DeGruy, F.V., Hahn, S.R., Linzer, M., Williams, J.B., Brody, D., Davies, M., 1997. Multisomatoform disorder: an alternative to undifferentiated somatoform disorder for the somatizing patient in primary care. Arch. Gen. Psychiatry 54, 352-358, http://dx.doi.org/10.1001/archpsyc. 1997.01830160080011.

Kroenke, K., Sharpe, M., Sykes, R., 2007. Revising the classification of somatoform disorders: key questions and preliminary recommendations. Psychosomatics 48, 277-285, http://dx.doi.org/10.1176/appi.psy.48.4.277.

Kroenke, K., 2003. Patients presenting with somatic complaints: epidemiology: psychiatric co-morbidity and management. Int. J. Methods Psychiatr. Res. 12, 34-43.

Kruglanski, A.W., 1989. The psychology of being right: the problem of accuracy in social perception and cognition. Psychol. Bull. 106, 395-409, http://dx.doi.org/ 10.1037/0033-2909.106.3.395.

Kveraga, K., Ghuman, A.S., Bar, M., 2007. Top-down predictions in the cognitive brain. Brain Cogn. 65, 145-168, http://dx.doi.org/10.1016/j.bandc.2007.06.007.

Landgrebe, M., Barta, W., Rosengarth, K., Frick, U., Hauser, S., Langguth, B. Rutschmann, R., Greenlee, M.W., Hajak, G., Eichhammer, P., 2008. Neuronal correlates of symptom formation in functional somatic syndromes: a fMRI study. Neuroimage 41, 1336-1344, http://dx.doi.org/10.1016/j.neuroimage. 2008.04.171.

Leventhal, H., Leventhal, E.A., 1993. Affect, cognition, and symptom perception. In: Foley, K.M., Chapman, C.R. (Eds.), Current and Emerging Issues in Cancer Pain: Research and Practice. Raven Press, pp. 153-173.

Leventhal, H., Diefenbach, M., Leventhal, E.A., 1992. Illness cognition: using common sense to understand treatment adherence and affect cognition interactions. Cognit. Ther. Res. 16, 143-163, http://dx.doi.org/10.1007/ BF01173486.

Leventhal, H., Leventhal, E.A., Contrada, R.J., 1998. Self-regulation, health, and behavior: a perceptual-cognitive approach. Psychol. Health 13, 717-733, http://dx.doi.org/10.1080/08870449808407425.

Lim, S.L., Kim, J.H., 2005. Cognitive processing of emotional information in depression, panic, and somatoform disorder. J. Abnorm. Psychol. 114, 50 http://dx.doi.org/10.1037/0021-843X.114.1.50.

Liston, C., McEwen, B.S., Casey, B.J., 2009. Psychosocial stress reversibly disrupts prefrontal processing and attentional control. Proc. Natl. Acad. Sci. U. S. A. 106, 912-917 http://dx.doi.org/10.1073/pnas.0807041106.

Lynn, S.K., Barrett, L.F., 2014. Utilizing signal detection theory. Psychol. Sci. 25, 1663-1673, http://dx.doi.org/10.1177/0956797614541991.

Müllerová, H., Lu, C., Li, H., Tabberer, M., 2014. Prevalence and burden of breathlessness in patients with chronic obstructive pulmonary disease managed in primary care. PLoS One 9 (1), e85540.
Marcus, D.K., Gurley, J.R., Marchi, M.M., Bauer, C., 2007. Cognitive and perceptual variables in hypochondriasis and health anxiety: a systematic review. Clin. Psychol. Rev. 27, 127-139, http://dx.doi.org/10.1016/i.cpr.2006.09.003.

Martin, M., Alexeeva, I., 2010. Mood volatility with rumination but neither attentional nor interpretation biases in chronic fatigue syndrome. Br. J. Health Psychol. 15, 779-796, http://dx.doi.org/10.1348/135910709x480346.

Martin, M., Chapman, S.C., 2010. Cognitive processing in putative functional gastrointestinal disorder: rumination yields orientation to social threat not pain. Eur. J. Pain 14, 207-213, http://dx.doi.org/10.1016/j.ejpain.2009.04.008.

Montoya, P., Sitges, C., García-Herrera, M., Izquierdo, R., Truyols, M., Blay, N., Collado, D., 2005. Abnormal affective modulation of somatosensory brain processing among patients with fibromyalgia. Psychosom. Med. 67, 957-963.

Mussgay, L., Klinkenberg, N., Rüddel, H., 1999. Heart beat perception in patients with depressive, somatoform, and personality disorders. J. Psychophysiol. 13, 27-36, http://dx.doi.org/10.1027//0269-8803.13.1.27.

Nakao, M., Barsky, A.J., 2007. Clinical application of somatosensory amplification in psychosomatic medicine. Biopsychosoc. Med. 1, 17, http://dx.doi.org/10.1186/ 1751-0759-1-17.

Naliboff, B.D., Berman, S., Chang, L., Derbyshire, S.W., Suyenobu, B., Vogt, B.A., Mayer, E.A., 2003. Sex-related differences in IBS patients: central processing of visceral stimuli. Gastroenterology 124 (7), 1738-1747.

Nijs, J., Meeus, M., Van Oosterwijck, J., Ickmans, K., Moorkens, G., Hans, G., De Clerck, L.S., 2012. In the mind or in the brain? Scientific evidence for central sensitisation in chronic fatigue syndrome. Eur. J. Clin. Invest. 42, 203-212, http://dx.doi.org/10.1111/j.1365-2362.2011.02575.x.

Norman, G.J., Berntson, G.G., Cacioppo, J.T., 2014. Emotion, somatovisceral afference, and autonomic regulation. Emotion Rev. 6 (2), 113-123.

Paulus, M.P., Stein, M.B., 2006. An insular view of anxiety. Biol. Psychiatry 60, 383-387, http://dx.doi.org/10.1016/j.biopsych.2006.03.042.

Pennebaker, I.W., Roberts, T.A., 1992. Toward a his and hers theory of emotion: gender differences in visceral perception. J. Soc. Clin. Psychol. 11 (3), 199

Pennebaker, J.W., 1982. The Psychology of Physical Symptoms. Springer, New York.

Pennebaker, J.W., 1995. Beyond laboratory-based cardiac perception: ecological interoception. In: Vaitl, D., Schandry, R. (Eds.), From the Heart to the Brain. The Psychophysiology of Circulation - Brain Interaction. Peter Lang, Frankfurt, pp. 389-406.

Petersen, S., van den Berg, R., Janssens, T., Van den Bergh, O., 2011. Illness and symptom perception: a theoretical approach towards an integrative measurement model. Clin. Psychol. Rev. 31, 428-439, http://dx.doi.org/10. 1016/j.cpr.2010.11.002.

Petersen, S., Schroijen, M., Mölders, C., Zenker, S., Van den Bergh, O., 2014. Categorical interoception perceptual organization of sensations from inside. Psychol. Sci. 25, 1059-1066, http://dx.doi.org/10.1177/0956797613519110.

Petersen, S., von Leupoldt, A., Van den Bergh, O., 2015a. Interoception and the uneasiness of the mind: affect as perceptual style. Front. Psychol. 6, 1408, http://dx.doi.org/10.3389/fpsyg.2015.01408.

Petersen, S., Van Staeyen, K., von Leupoldt, A., Vögele, C., Van den Bergh, O., 2015b. Interoception and symptom reporting: disentangling accuracy and response bias. Front. Psychol.: Conscious. Res. 6, 732, http://dx.doi.org/10.3389/fpsyg. 2015.00732.

Petersen, S., Vögele, C., \& Van den Bergh, O., (submitted). The Procrustes Effect: How anxiety modifies the perception of bodily sensation.

Posserud, I., Svedlund, J., Wallin, J., Simrén, M., 2009. Hypervigilance in irritable bowel syndrome compared with organic gastrointestinal disease. J. Psychosom. Res. 66, 399-405, http://dx.doi.org/10.1016/i.jpsychores.2008.09.020.

Powell, D.J., Liossi, C., Moss-Morris, R., Schlotz, W., 2013. Unstimulated cortisol secretory activity in everyday life and its relationship with fatigue and chronic fatigue syndrome: a systematic review and subset meta-analysis. Psychoneuroendocrinology 38, 2405-2422.

Price, D.D., Riley, J.L., Wade, J.B., 2001. Psychophysical approaches to measurement of the dimensions and stages of pain. In: Turk, D.C., Melzack, R. (Eds.), Handbook of Pain Assessment. , 2nd ed. Guilford Press, New York, NY US, pp. 53-75.

Rief, W., Barsky, A.I., 2005. Psychobiological perspectives on somatoform disorders. Psychoneuroendocrinology 30, 996-1002.

Rief, W., Broadbent, E., 2007. Explaining medically unexplained symptoms-models and mechanisms. Clin. Psychol. Rev. 27, 821-841, http://dx.doi.org/10.1016/j. cpr.2007.07.005.

Rief, W., Martin, A., 2014. How to use the new DSM-5 somatic symptom disorder diagnosis in research and practice: a critical evaluation and a proposal for modifications. Annu. Rev. Clin. Psychol. 10, 339-367, http://dx.doi.org/10. 1146/annurev-clinpsy-032813-153745.

Rief, W., Hiller, W., Margraf, I., 1998. Cognitive aspects of hypochondriasis and the somatization syndrome. J. Abnorm. Psychol. 107, 587.

Robbins, J.M., Kirmayer, L.J., 1991. Attributions of common somatic symptoms. Psychol. Med. 21, 1029-1045, http://dx.doi.org/10.1017/S0033291700030026.

Roberts, T.A., Pennebaker, J.W., 1994. Gender differences in perceiving internal state: toward a his-and-hers model of perceptual cue use. Adv. Exp. Soc. Psychol. 27, 143.

Robinson, O.J., Vytal, K., Cornwell, B.R., Grillon, C., 2013. The impact of anxiety upon cognition: perspectives from human threat of shock studies. Front. Hum. Neurosci. 7, 203, http://dx.doi.org/10.3389/fnhum.2013.00203.

Ryan, C.M., Dulay, D., Suprasongsin, C., Becker, D.J., 2002. Detection of symptoms by adolescents and young adults with type 1 diabetes during experimental enduction of mild hypoglycemia role of hormonal and psychological variables. Diab.Care 25, 852-858. 
Schaefer, M., Egloff, B., Witthöft, M., 2012. Is interoceptive awareness really altered in somatoform disorders? Testing competing theories with two paradigms of heartbeat perception. J. Abnormal Psychol. 121, 719-724, http://dx.doi.org/10. 1037/a0028509.

Schaefer, M., Egloff, B., Gerlach, A.L., Witthöft, M., 2014. Improving heartbeat perception in patients with medically unexplained symptoms reduces symptom distress. Biol. Psychol. 101, 69-76, http://dx.doi.org/10.1016/j. biopsycho.2014.05.012.

Schandry, R., Leopold, C., Vogt, M., 1996. Symptom reporting in asthma patients and insulin-dependent diabetics. Biol. Psychol. 42, 231-244, http://dx.doi.org/ 10.1016/0301-0511(95)05157-0.

Schreiber, F., Neng, I.M., Heimlich, C., Witthöft, M., Weck, F., 2014. Implicit affective evaluation bias in hypochondriasis: findings from the Affect Misattribution Procedure. J. Anxiety Disord. 28, 671-678, http://dx.doi.org/10.1016/j.janxdis. 2014.07.004.

Schroeder, S., Gerlach, A.L., Martin, A., 2014. Implicit affective evaluation of somatosensory sensations in patients with noncardiac chest pain. J. Behav. Ther. Exp. Psychiatry 45, 381-388, http://dx.doi.org/10.1016/j.jbtep.2014.04. 002.

Schulz, A., Vögele, C., 2015. Interoception and stress. Front. Psychol. 6, 993, http:// dx.doi.org/10.3389/fpsyg.2015.00993.

Schwille-Kiuntke, J., Mazurak, N., Enck, P., 2015. Systematic review with meta-analysis: post-infectious irritable bowel syndrome after travellers' diarrhoea. Alimentary Pharmacol. Ther. 41, 1029-1037, http://dx.doi.org/10. 1111/apt.13199.

Sears, S.F., Serber, E.R., Alvarez, L.G., Schwartzman, D.S., Hoyt, R.H., Ujhelyi, M.R., 2005. Understanding atrial symptom reports: objective versus subjective predictors. Pacing Clin. Electrophysiol. 28, 801-807.

Seger, C.A., Peterson, E.J., 2013. Categorization=decision making + generalization. Neurosci. Biobehav. Rev. 37, 1187-1200, http://dx.doi.org/10.1016/i. neubiorev.2013.03.015.

Seth, A.K., Suzuki, K., Critchley, H.D., 2011. An interoceptive predictive coding model of conscious presence. Front. Psychol. 2, 395, http://dx.doi.org/10.3389/ fpsyg.2011.00395.

Seth, A.K., 2013. Interoceptive inference, emotion, and the embodied self. Trends Cogn. Sci. 17, 565-573, http://dx.doi.org/10.1016/j.tics.2013.09.007.

Stein, M.B., Simmons, A.N., Feinstein, J.S., Paulus, M.P., 2007. Increased amygdala and insula activation during emotion processing in anxiety-prone subjects. Am. J. Psychiatry 164, 318-327 http://dx.doi.org/10.1176/appi.ajp.164.2.318.

Strickberger, S.A., Ip, J., Saksena, S., Curry, K., Bahnson, T.D., Ziegler, P.D., 2005. Relationship between atrial tachyarrhythmias and symptoms. Heart Rhythm 2 125-131, http://dx.doi.org/10.1016/j.hrthm.2004.10.042.

Tak, L.M., Rosmalen, J.G., 2010. Dysfunction of stress responsive systems as a risk factor for functional somatic syndromes. J. Psychosom. Res. 68 (5), 461-468.

Tak, L.M., Riese, H., de Bock, G.H., Manoharan, A., Kok, I.C., Rosmalen, J.G., 2009. As good as it gets? A meta-analysis and systematic review of methodological quality of heart rate variability studies in functional somatic disorders. Biol. Psychol. 82, 101-110, http://dx.doi.org/10.1016/j.biopsycho.2009.05.002.

Tak, L.M., Cleare, A.I., Ormel, I., Manoharan, A., Kok, I.C., Wessely, S., Rosmalen, I.G. 2011. Meta-analysis and meta-regression of hypothalamic-pituitary-adrenal axis activity in functional somatic disorders. Biol. Psychol. 87, 183-194, http:// dx.doi.org/10.1016/i.biopsycho.2011.02.002.

Tillisch, K., Mayer, E.A., Labus, J.S., 2011. Quantitative meta-analysis identifies brain regions activated during rectal distension in irritable bowel syndrome. Gastroenterology 140, 91-100, http://dx.doi.org/10.1053/i.gastro.2010.07.053.

Tsakiris, M., Tajadura-Jiménez, A., Costantini, M., 2011. Just a heartbeat away from one's body: interoceptive sensitivity predicts malleability of body-representations. Proc. R. Soc. Lond. B: Biol. Sci. 278 (1717), 2470-2476, http://dx.doi.org/10.1098/rspb.2010.2547.

Turner, D., Griffiths, A.M., Mack, D., Otley, A.R., Seow, C.H., Steinhart, A.H. Silverberg, M.S., Hyams, I., Guvatt, G.H., 2010. Assessing disease activity in ulcerative colitis: patients or their physicians? Inflamm. Bowel Dis. 16, 651-656, http://dx.doi.org/10.1002/ibd.21088.

Van Cauwenbergh, D., Nijs, I., Kos, D., Weijnen, L., Struyf, F., Meeus, M., 2014 Malfunctioning of the autonomic nervous system in patients with chronic fatigue syndrome: a systematic literature review. Eur. J. Clin. Invest. 44, 516-526.

Van Damme, S., Van Hulle, L., Spence, C., Devulder, J., Brusselmans, G., Crombez, G., 2014. Hypervigilance for innocuous tactile stimuli in patients with fibromvalgia: an experimental approach. Eur. J. Pain 19, 706-714, http://dx. doi.org/10.1002/ejp.593.

Van De Cruys, S., 2014. To Err and Err, but Less and Less. Predictive Coding and Affective Value in Perception, Art, and Autism. Doctoral Dissertation. KU Leuven.

Van Dessel, N., Den Boeft, M., van der Wouden, J.C., Kleinstäuber, M., Leone, S.S., Terluin, B., Numans, M.E., van der Horst, H.E., van Marwijk, H., 2014. Non-pharmacological interventions for somatoform disorders and medically unexplained physical symptoms (MUPS) in adults. Cochrane Database Syst. Rev. 11, CD011142, http://dx.doi.org/10.1002/14651858.cd011142.pub2.
Van Oudenhove, L., Aziz, Q., 2013. The role of psychosocial factors and psychiatric disorders in functional dyspepsia. Nat. Rev. Gastroenterol. Hepatol. 10, 158-167, http://dx.doi.org/10.1038/nrgastro.2013.10.

Van Oudenhove, L., Vandenberghe, J., Geeraerts, B., Vos, R., Persoons, P., Fischler, B., Demyttenaere, K., Tack, J., 2008. Determinants of symptoms in functional dyspepsia: gastric sensorimotor function, psychosocial factors or somatisation? Gut 57, 1666-1673, http://dx.doi.org/10.1136/gut.2008.158162.

Van den Bergh, O., Kempynck, P.J., Van de Woestijne, K.P., Baeyens, F., Eelen, P., 1995. Respiratory learning and somatic complaints: a conditioning approach using CO2-enriched air inhalation. Behav. Res. Ther. 33, 517-527, http://dx.doi. org/10.1016/0005-7967(94)00080-4.

Van den Bergh, O., Stegen, K., Van de Woestijne, K.P., 1997. Learning to have psychosomatic complaints: conditioning of respiratory behavior and complaints in psychosomatic patients. Psychosom. Med. 59, 13-23.

Van den Bergh, O., Winters, W., Devriese, S., Van Diest, I., 2002. Learning subjective health complaints. Scand. J. Psychol. 43, 147-152, http://dx.doi.org/10.1111/ 1467-9450.00280.

Vandenbroucke, S., Crombez, G., Harrar, V., Brusselmans, G., Devulder, I., Spence, C. Goubert, L., 2014. Fibromyalgia patients and controls are equally accurate in detecting tactile stimuli while observing another in pain: an experimental study. Attention Percept. Psychophys. 76, 2548-2559, http://dx.doi.org/10. 3758/s13414-014-0729-9.

World Health Organization, 1992. The ICD-10 classification of mental and behavioural disorders: clinical descriptions and diagnostic guidelines. Geneva: World Health Organization.

Walentynowicz, M., Raes, F., Van Diest, I., Van den Bergh, O., 2016. The specificity of health-related autobiographical memories in patients with Somatic Symptom Disorder. Psychosom. Med., http://dx.doi.org/10.1097/PSY. 0000000000000357 , in press.

Watson, D., Pennebaker, I.W., 1989. Health complaints, stress: and distress: exploring the central role of negative affectivity. Psychol. Rev. 96, 234-254.

Wessely, S., White, P.D., 2004. In debate: there is only one functional somatic syndrome. Brit. J. Psychiatry 185, 95-96, http://dx.doi.org/10.1192/bjp.185.2. 95.

Wessely, S., Nimnuan, C., Sharpe, M., 1999. Functional somatic syndromes: one or many? Lancet 354, 936-939 http://dx.doi.org/10.1016/S0140-6736(98)083202 .

Wiech, K., 2016. Deconstructing the sensation of pain: the influence of cognitive processes on pain perception. Science 354, 584-587, http://dx.doi.org/10 $1126 /$ science.aaf8934

Witthöft, M., Hiller, W., 2010. Psychological approaches to origins and treatments of somatoform disorders. Annu. Rev. Clin. Psychol. 6, 257-283, http://dx.doi. org/10.1146/annurev.clinpsy.121208.131505.

Witthöft, M., Gerlach, A., Bailer, J., 2006. Selective attention, memory bias, and symptom perception in idiopathic environmental intolerance and somatoform disorders. J. Abnorm. Psychol. 115, 397-407, http://dx.doi.org/10.1037/0021843X.115.3.397.

Witthöft, M., Basfeld, C., Steinhoff, M., Gerlach, A.L., 2012. Can't suppress this feeling: automatic negative evaluations of somatosensory stimuli are related to the experience of somatic symptom distress. Emotion 12, 640-649, http:/ dx.doi.org/10.1037/a0024924.

Witthöft, M., Fischer, S., Jasper, F., Rist, F., Nater, U.M., 2016. Clarifying the latent structure and correlates of somatic symptom distress: a bifactor model approach. Psychol. Assess. 28 (109), 15, http://dx.doi.org/10.1037/pas0000150.

Woud, M.L., Zhang, X.C., Becker, E.S., Zlomuzica, A., Margraf, I., 2016. Catastrophizing misinterpretations predict somatoform-related symptoms and new onsets of somatoform disorders. J. Psychosom. Res. 81, 31-37.

Wright, C.E., Ebrecht, M., Mitchell, R., Anggiansah, A., Weinman, I., 2005. The effect of psychological stress on symptom severity and perception in patients with gastro-oesophageal reflux. J. Psychosom. Res. 59, 415-424, http://dx.doi.org/ 10.1016/i.jpsychores.2005.05.012.

Yiend, J., 2010. The effects of emotion on attention: a review of attentional processing of emotional information. Cognit. Emotion 24, 3-47, http://dx.doi org/10.1080/02699930903205698.

Zaki, J., Davis, J.I., Ochsner, K.N., 2012. Overlapping activity in anterior insula during interoception and emotional experience. Neuroimage 62, 493-499, http://dx.doi.org/10.1016/i.neuroimage.2012.05.012.

Zaman, J., Vlaeyen, J.W., Van Oudenhove, L., Wiech, K., Van Diest, I., 2015. Associative fear learning and perceptual discrimination: a perceptual pathway in the development of chronic pain. Neurosci. Biobehav. Rev. 51, 118-125, http://dx.doi.org/10.1016/i.neubiorev.2015.01.009.

Zikopoulos, B., Barbas, H., 2006. Prefrontal projections to the thalamic reticular nucleus form a unique circuit for attentional mechanisms. J. Neurosci. 26 (28), 7348-7361

van der Veek, S.M., Derkx, B.H., Plak, R.D., Benninga, M.A., Boer, F., Lindauer, R.J., de Haan, E., 2014. Attentional bias to activity of different parts of the body in children with functional abdominal pain: an experimental study. J. Pediatr. Psychol. 39, 438-449, http://dx.doi.org/10.1093/jpepsy/jsu004. 